\title{
Resilience of Perceptual Metacognition in a
}

\section{Dual-Task Paradigm}

Mahiko Konishi ${ }^{12}$, Clémence Compain ${ }^{12}$, Bruno Berberian ${ }^{3}$, Jérôme Sackur ${ }^{14}$, Vincent de

\author{
Gardelle $^{5}$
}

\author{
1) Laboratoire de Sciences Cognitives et de Psycholinguistique, Dept d'Etudes Cognitives, ENS, PSL University, EHESS, CNRS, \\ Paris, France. \\ 2) Centre d'Economie de la Sorbonne, CNRS \& Université Paris 1, Paris, France. \\ 3) ONERA, The French Aerospace Lab, Information Processing and Systems Department, 13661, Salon Cedex Air, France. \\ 4) École Polytechnique, Palaiseau, France. \\ 5) Paris School of Economics and CNRS, France.
}

\begin{abstract}
When people do multiple tasks at the same time, it is often found that their performance is worse relative to when they do those same tasks in isolation. However, one aspect that has received little empirical attention, is whether the ability to monitor and evaluate one's task performance is also affected by multitasking. How does dual-tasking affect metacognition and its relation to performance? We investigated this question through the use of a visual dual-task paradigm with confidence judgments. Participants categorized both the color and the motion direction of moving dots, and then rated their confidence in both responses. Across four experiments, participants $(\mathrm{N}=87)$ exhibited a clear dual-task cost at the perceptual level, while no cost at the
\end{abstract}


metacognitive level. We discuss this resilience of metacognition to multitasking costs, and examine how our results fit onto current models of perceptual metacognition.

Keywords: metacognition; confidence; multitasking; dual-task

Wordcount: 2898 


\section{Introduction}

Imagine an aircraft pilot encountering a storm during a flight, just as they close in to their destination airport. As they scramble to monitor different gauges, communicate via radio with air traffic control, and keep the plane steady, they make a crucial mistake. Is the aircraft pilot unaware of their mistake, precisely because of the multitasking situation they're in? In other words, how is the ability to evaluate one's own performance affected when we have to perform and monitor multiple tasks at the same time? This is the question that we set out to answer in the current investigation.

It has long been known that people can report feelings of confidence that correlate with their objective performance in tasks (Henmon, 1911; Metcalfe \& Shimamura, 1994; Nelson \& Narens, 1990; Peirce \& Jastrow, 1884). In other words, people are able to perform a second-order task (Type 2 task, e.g. a confidence rating), on top of a first-order task (Type 1 task, e.g. a perceptual decision). This second-order cognitive monitoring of first-order processes, also known as metacognition, has been shown to play a crucial role in informing future decisions (Folke et al., 2017) and in guiding learning (Boldt et al., 2019; Guggenmos et al., 2016; Hainguerlot et al., 2018). As these feelings of confidence seem to be computed at an abstract, task-independent level (de Gardelle et al., 2016; de Gardelle \& Mamassian, 2014), it is now considered that metacognition involves a higher-order system evaluating information coming from lower, first-order systems (Fleming \& Daw, 2017; Maniscalco \& Lau, 2016), and relying on prefrontal areas of the brain (Fleming et al., 2010, 2012; Fleming \& Dolan, 2012).

People often have trouble performing even two simple tasks at the same time (Pashler, 1994) in particular when these two tasks engage the same resources, either peripheral or central (Salvucci \& Taatgen, 2008; Wickens, 2002). Surprisingly, despite the growing interest in metacognition in decision making, there has been only a handful of studies assessing how individuals evaluate their performance in dual-task conditions (Finley et al., 2014; Horrey et al., 2009; Maniscalco \& Lau, 2015; Sanbonmatsu et al., 2016). In these studies, participants had to evaluate their 
performance in a primary task while also doing a secondary task, such as evaluating how driving is affected by phone usage (Sanbonmatsu et al., 2016), or how visual discrimination is affected by working memory (Maniscalco \& Lau, 2015). In particular, Maniscalco \& Lau (2015) reasoned that as both metacognition and working memory solicit overlapping brain networks, engaging in a concurrent working memory task should impair perceptual metacognition, which is indeed what they found.

However, as these studies only used one metacognitive task, the potential cost of performing two concurrent metacognitive tasks remains unknown. One reason to expect that dual-task costs at the metacognitive level is that metacognition is thought to rely on central processes, which have shown to be limited from a processing bottleneck (Collette et al., 2005; Dux et al., 2006; Sigman \& Dehaene, 2005; Szameitat et al., 2002). In other words, performing and monitoring multiple tasks concurrently might not only degrade performance in Type 1 tasks, it may also deteriorate Type 2 evaluations, over and above the expected impact that a loss in performance has on metacognition. This would lead to a potentially catastrophic scenario: not only does the pilot make more errors, they also become less able to detect these errors.

The aforementioned studies suggest that when two tasks are to be performed concurrently, metacognition is degraded. Here, we wanted to analyse the impact of multitasking on perceptual metacognition in the simplest case, where two equally important tasks are to be performed concurrently and based on the same visual stimulus. In a way, this situation maximizes the overlap between the two tasks (Wickens, 2002), increasing the chances to obtain a cost at the metacognitive level too. We thus designed a visual dual-task paradigm, using a random dot kinematogram stimulus that supports two orthogonal tasks. In this task, participants perform blocks of either a motion or a color discrimination task in isolation, or they performed both perceptual tasks together, resulting in a 2 (task: color or motion) x 2 (condition: single or dual) within-subject experimental design. Each perceptual judgment is followed by a confidence judgment. The stimuli and experimental design are shown in Figure 1. 


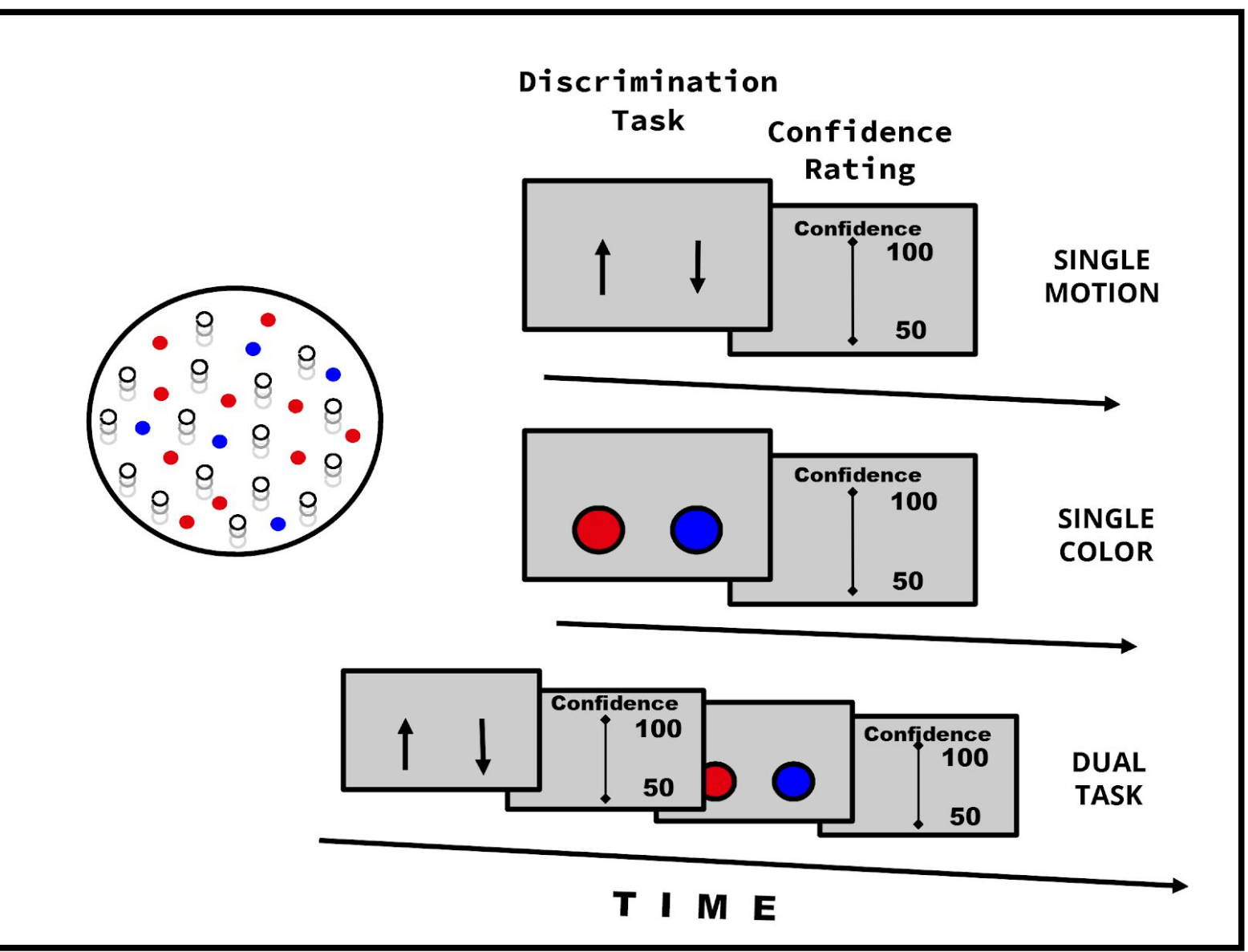

Figure 1 - Dual-Task Paradigm

Participants were presented with a random dot kinematogram (shown on the left), and then proceeded to give a perceptual discrimination response, followed by a confidence rating (on a scale from 50 to 100) on that response. On each trial there were either more blue or red dots (color task), while the white dots moved either upwards or downwards (motion task) with a certain coherence. The design was blocked, so that participants did either 30-trial blocks of motion discrimination, color numerosity discrimination, or both (dual-task condition, shown at the bottom). For the dual-task condition, the order of the responses for the two tasks was counterbalanced across blocks.

We report four experiments conducted with these stimuli and tasks (see Table 1 in the Methods section for a summary). Our first two experiments both used an adaptive staircase procedure, but with different stimulus duration (1500 and $300 \mathrm{~ms}$ ) so as to vary task demands and create a time pressure that would minimize the chance that participants performed the two tasks serially instead of concurrently. The results from these first two experiments were surprising: we found 
that on average, metacognitive efficiency did not degrade in the dual-task condition (it rather improved in one task). To evaluate whether this surprising result would replicate, we conducted two additional experiments with short duration stimuli, one using a staircase method and one using constant stimuli. Using constant stimuli ensures that participants are given the same amount of perceptual information in the single and dual conditions, though their ability to use this information may differ due to dual-tasking costs. Across all four experiments, we found no evidence for a dual-task cost in terms of metacognitive efficiency, despite a clear dual-task cost at the Type 1 level. 


\section{Methods}

\section{Participants}

The first two experiments (long duration / staircase procedure and short duration / staircase procedure) were conducted at the Laboratory of Experimental Economics in Paris (LEEP), in the Maison des Sciences Économiques of Université Paris 1 - Panthéon-Sorbonne, where participants were tested in a large room with 20 individual computers, while the two other experiments (short/staircase replication and short/constant stimuli) were conducted at the Département d'Études Cognitives of the École Normale Supérieure, where participants were tested individually in isolated booths. Participants were recruited from the volunteer databases of the LEEP and of the Relais d'Information en Sciences de la Cognition (RISC) unit, which is largely composed of French students. Each session lasted between 60 and 90 minutes. Ninety-three participants in total (between 18 and 38 years old; 63 women) took part in this study (see Table 1). Participants in the first two experiments were paid a fixed rate plus a bonus depending on a combination of their Type 1 and Type 2 performance: this was calculated using a matching probability rule that was explained to participants beforehand, and which has been shown to be the best elicitator of people's subjective feelings of confidence (Hollard et al., 2016; Karni, 2009). They were paid on average $14 €$. Participants in the last two experiments were paid a fixed rate of $20 €$.

\section{Stimuli and Tasks}

The stimuli and tasks were programmed using Psychtoolbox 3.0.14 (Kleiner et al., 2007) in MATLAB 9.4.0 (R2018a). The main stimulus used in all experiments was a random-dot kinematogram (RDK) consisting of two hundred dots (radius of 0.2 deg each approximately with a distance from the screen of $\sim 60 \mathrm{~cm}$, with a finite lifetime of $167 \mathrm{~ms}$ ) presented in a circular area (radius of $5^{\circ}$ approximately) in the center of the screen. One hundred of these dots were white, and these were used for the motion discrimination task: these dots moved with a speed of 1.2 
$\mathrm{deg} / \mathrm{s}$ either upwards or downwards with a coherence which varied on a trial-to-trial basis in the staircase experiments, or was determined after a calibration phase and then fixed throughout the experiment in the constant stimuli experiment. Participants had to discriminate if the dots were moving either upwards or downwards on each trial, and gave their response using two keys ("D" for upward, "F" for downwards). The other hundred dots were used for the color task: these always moved without coherence with a speed of $0.3 \mathrm{deg} / \mathrm{s}$. These dots were either red or blue in a certain ratio, which again changed on a trial-to-trial basis in the staircase experiments, or was determined after a calibration phase and then fixed throughout the experiment in the constant stimuli experiment. Participants had to discriminate if there were more blue or red dots on the screen, on every trial, and responded using two keys ("E" for blue, "R" for red). After each discrimination trial (the Type 1 task), participants rated their confidence on their decision using the mouse cursor on a vertical scale ranging from 100 ("I'm sure I made the correct decision") to 50 ("I responded randomly"). For the dual-task condition, the order of the responses for the two tasks was counterbalanced across blocks. The raw data, the tasks' stimuli, and the scripts used for analyses are available at https://osf.io/e2jpz/.

\section{Procedure and Instructions to Participants}

Participants signed a consent form and were then instructed on the nature of the Type 1 (the

discrimination tasks) and Type 2 (the confidence ratings) tasks; furthermore, they were encouraged to reflect on their subjective feelings of confidence in their Type 1 decisions and to try to use the whole range of the confidence scale throughout the experiment. They then completed a training session which consisted of three blocks (a single-color, a single-motion, and a dual-task block each) of 30 trials each, for which the staircase procedure was already implemented: in the actual experimental session, the stimulus level started from where it left off in the training session.

\section{Experimental Design}

All the experiments followed a simple 2 (task) x 2 (condition) within-subject, blocked design. Participants performed blocks of 30 trials; there were three block types: single color, single 
movement, or dual-task condition. There were 18 blocks, for a total of 540 trials (180 per condition). The 18 blocks were divided into six macro-blocks, within which blocks of all three block types were presented to participants once. The order of presentation of conditions in the macro-blocks was randomised across participants.

The four experiments presented here all follow the same design and procedure. The only differences were the duration of stimulus presentation, and the procedure used to vary the stimulus difficulty throughout the task. In one study the main stimulus was presented for $1500 \mathrm{~ms}$ (long duration experiment), and in the other three studies for $300 \mathrm{~ms}$ (short duration experiments). Likewise, three studies used an adaptive staircase procedure, while the last one used constant stimuli. The first two experiments (long duration and short duration staircase) had unique subjects participating in them, while the same cohort of participants performed the replication and the constant stimuli experiments: they completed the staircase experiment in the first session, after which their psychometric curves were fitted to their response data in order to estimate the appropriate stimulus level to elicit $78 \%$ correct performance. This was then used in the constant stimulus experiment, for which participants returned for a second session between one and four days after the first one.

\section{Staircase Procedure and Constant Stimuli}

In all staircase experiments we used a 1-up-1-down staircase with unequal step sizes: step size was of $4 \%$ the maximum value of the stimulus, and the ratio of down-step/up-step was 0.2845 , which results in the staircase converging to $78 \%$ accuracy (García-Pérez, 1998). In the constant stimuli experiment, stimulus difficulty for both tasks was estimated using participants' psychometric curves from the first staircase session, in order to achieve $\sim 78 \%$ accuracy in the single condition.

\section{Exclusion Criteria}

We used two exclusion criteria at the participant level. First we excluded participants who had over $90 \%$ accuracy or below $60 \%$ accuracy for Type 1 discrimination performance in any of the tasks or conditions. There are two reasons to do so: firstly, this is a sign that the staircase failed 
to converge for such participants. Secondly, it is problematic to study metacognition in participants that perform close to ceiling or floor level: because of the relationship between type 1 and type 2 information, the former will tend to have perfect metacognition, while the latter will show the opposite pattern. This resulted in the exclusion of eleven participants: these were mostly from the constant stimuli experiment, as using the same stimulus level for both singleand dual-task conditions raised the chances that participants performed either too close to ceiling or floor level, in one of the two conditions. We also excluded participants that never varied their Type 2 confidence response (for example by always responding " 100 "): this is a necessary step because with no variability in the confidence responses, a measure of metacognitive efficiency cannot be reliably computed. We thus looked at the distribution of the standard deviations of participants' confidence responses in the two tasks and conditions, and decided to exclude participants with a standard deviation lower than 5 in any task or condition (see Figure $G$ in section 3 Supplementary Plots of the Appendix). This resulted in the exclusion of an additional eight participants.

After applying these exclusion criteria, we analysed data from eighty-seven participants (thirteen performed well in the short/staircase replication, but did not pass the exclusion criteria in the short/constant experiment, and their data for the latter was consequently removed and not analysed), eighteen of which took part in both of the last two experiments. A summary of the experiments and participants is available in Table 1. Finally, at the trial level we excluded every first trial of each new block (for task-switching costs), and every trial for which the log value of the response time was above or below 2.5 times the standard deviation of the log-RT distribution $(\sim 2.5 \%$ of all trials $)$.

\begin{tabular}{|c|c|c|c|c|c|c|}
\hline Experiment & $\begin{array}{c}\text { Stimulus } \\
\text { Duration }\end{array}$ & Procedure & $\begin{array}{c}\text { Initial } \\
\text { Participants }\end{array}$ & $\begin{array}{c}\text { \# Excluded For } \\
\text { Ceiling/Floor } \\
\text { Performance }\end{array}$ & $\begin{array}{c}\text { \# Excluded For } \\
\text { Confidence } \\
\text { Variance }\end{array}$ & $\begin{array}{c}\text { Participants } \\
\text { Analysed }\end{array}$ \\
\hline 1 & $1500 \mathrm{~ms}$ & Staircase & 30 & 0 & 3 & 27 \\
\hline
\end{tabular}




\begin{tabular}{|c|c|c|c|c|c|c|}
\hline 2 & $300 \mathrm{~ms}$ & Staircase & 30 & 1 & 2 & 27 \\
\hline 3 & $300 \mathrm{~ms}$ & Staircase & 33 & 0 & 0 & 33 \\
\cline { 1 - 4 } & $300 \mathrm{~ms}$ & $\begin{array}{c}\text { Constant } \\
\text { Stimuli }\end{array}$ & 31 & 7 & 6 & 18 \\
\hline
\end{tabular}

Table 1 - Summary of Experiments and Participants

Thirty-one participants, the same that completed the short duration / staircase replication, also completed the short duration / constant experiment (two dropped out in between sessions).

\section{Statistical Analyses}

\section{Discrimination Performance}

For each participant, and for each condition, we fitted Cumulative Gaussian functions describing the proportion of responses indicating "upwards" responses in the motion task, and "red" responses in the color task, respectively as a function of motion coherence, and proportion of red dots relative to blue dots (see Figure F in section 3 Supplementary Plots of the Appendix for an example). This was done with the quickpsy package (Linares \& López-Moliner, 2016) in the R environment (R Core Team, 2018). The mean and standard deviation were free parameters, while the lapse rate was set to a free parameter with an upper bound of $2 \%$ and a lower bound of $1 \%$. It is known that if lapses are not taken into account in the fitting process, it can bias both threshold and slope estimates (Wichmann \& Hill, 2001); however, letting the lapse rate vary freely can also induce biases (Prins, 2012): we thus chose these bounds to allow for some degree of variation, while keeping the estimates to lapses rates that are usually found in the field. After fitting, we extracted the slopes of the psychometric curves (the inverse of the standard deviation of the Cumulative Gaussians) as a measure of discrimination sensitivity. The goodness-of-fit was never unacceptably poor $(\mathrm{p}>.05$ of the deviance for all the participants, using bootstrapping; Linares \& López-Moliner, 2016) . 
The logic behind our four experiments was to understand if some specific experimental manipulation (such as stimulus duration, or the staircase procedures) could influence the cost (or the absence of a cost) at the metacognitive level. Given that the stimuli used (the RDK) and the experimental procedures were identical across experiments, we reasoned that the best way to analyse the effects of task and experimental condition (single vs dual) on discrimination and metacognitive performance, and to increase overall statistical power, was to run linear mixed models across our dataset. However, we included the factors (e.g. stimulus duration) that could have influenced performance, as well as having each experiment as a random effect. To analyse the effect of task type (motion vs. color), stimulus duration (300ms vs. 1500ms) and condition (single vs. dual) on participants' discrimination sensitivity, we ran linear mixed models with the R package lme4 (Bates et al., 2015). All other data manipulation and plotting was done with the tidyverse package (Wickham, 2017). Task type, condition, and duration were included in the model as fixed effects, while participants and experiments were included as random effects. Experimental procedure (staircase/constant stimuli) was initially also added to the models, but had no effect and was never selected as a factor in the best descripting models. P-values for fixed effects were calculated using the lmerTest (Kuznetsova et al., 2017) package which implements Satterthwaite's method to judge the significance of fixed effects. We then compared all possible models through the step function (which performs backward elimination of non-significant fixed and random effects) of the lmerTest package to find the best fitting model. A list of the best models tested for each analysis is available in Table Y, section 2 Linear Mixed Models of the Appendix. We also conducted post-hoc ANOVAs in order to better understand the significant interactions observed with these linear mixed models. Eta square and partial eta square were calculated using the sjStats package (Lüdecke, 2020). Bayes factors were calculated with the BayesFactor package (Morey \& Rouder, 2018).

\section{Metacognitive Efficiency}

The confidence ratings collected after every perceptual decision were analyzed to measure the efficiency of the metacognitive system. Metacognitive efficiency is measured by the M-ratio (Fleming \& Lau, 2014), which quantifies the ability of an observer to discriminate between 
correct responses and errors, relative to the information they have about the stimulus. Importantly, unlike other measures of metacognition, the M-ratio measure controls for the level of Type 1 performance in the task. This is crucial, as a decrease in Type 2 performance, such as one induced by multitasking, is to be expected following a decrease in Type 1 performance. The M-ratio can thus reveal the true impact of dual-tasking on metacognition. Metacognitive efficiency was computed for each participant, in each task and condition separately, using the metaSDT package (Craddock, 2018) in the R environment. In order to compute M-ratio, the continuous confidence ratings for each participant were median split onto high- and low-confidence, separately by task and condition (Maniscalco \& Lau, 2014). The analysis of the effects of task and condition on metacognitive efficiency was performed in a parallel manner to the one described for discrimination performance in the previous section, using linear mixed models. 


\section{Results}

\section{Discrimination Performance}

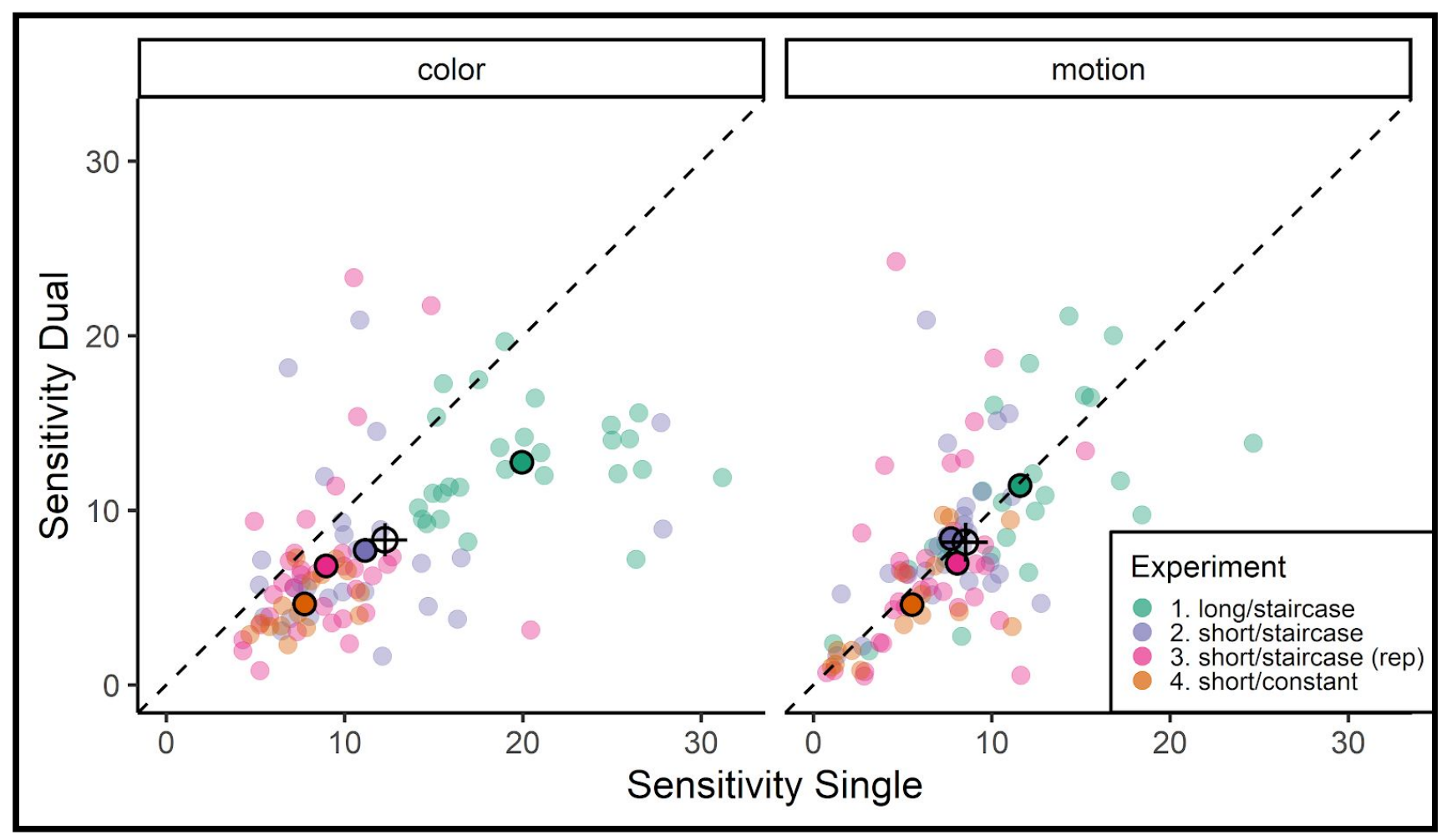

Figure 2 - Discrimination Performance

The figure shows participants' discrimination sensitivity (as measured by the slope of their psychometric curves) in the singleand dual-task conditions, for each task. The dashed diagonal lines show equality in sensitivity between single and dual conditions. A point below the dashed line means that the participant had better sensitivity in the single- than in the dual-task condition, i.e. they experienced a dual-tasking cost. Every point is one subject in one of the two tasks; colors refer to different experiments (see legend). Group averages for each experiment are shown with the black outlined, color filled shapes. Group averages for each task are shown with the black empty shapes; error bars within these shapes represent $95 \%$ confidence intervals.

Figure 2 shows the discrimination sensitivity values for each participant in the two conditions, separately for the two tasks. A dual-task cost can be seen in the figure for both tasks, as most 
participants showed increased sensitivity in the single-task condition; we tested for the statistical significance of this cost with linear mixed models. The best fitting model here had task, condition, stimulus duration, and their interactions as fixed effects, a random intercept for each participant, and a random intercept by experiment. This model (intercept $=12.7$, participant SD $=2.24$, experiment $\mathrm{SD}=1.29$ ) revealed the following effects of interest: a significant effect of condition, so that the dual-task condition predicted lower discrimination sensitivity ( $\square_{\text {single }}=$ $7.18,95 \%$ CI $[4.73,9.62], \mathrm{t}=5.73, \mathrm{p}<.001)$; a significant effect of stimulus duration $\left(\square_{\text {short }}=\right.$ $-6.22,95 \%$ CI $[-9.53,-2.92], \mathrm{t}=3.29, \mathrm{p}=.043)$, so that the longer duration predicted higher sensitivity; a significant interaction between task and condition, to the effect that the dual-task cost on sensitivity was increased in the color task $\left(\square_{\text {motionXsingle }}=-7.01,95 \%\right.$ CI $[-10.46,-3.55]$, $\mathrm{t}$ $=3.95, \mathrm{p}<.001)$. To further understand the interaction between task and condition, a post-hoc repeated measure ANOVA with condition as within-subject factor was ran separately in each task, suggesting that this dual-task cost in discrimination sensitivity was evident in the color task $\left(F(1,86)=45.88, p<.001, \eta^{2}=.108\right.$, partial $\left.\eta^{2}=.765\right)$, but not in the motion task $(F(1,86)=0.23$ $\left., \mathrm{p}=.634, \mathrm{BF}_{0}=6.06\right)$. 


\section{Response Times}

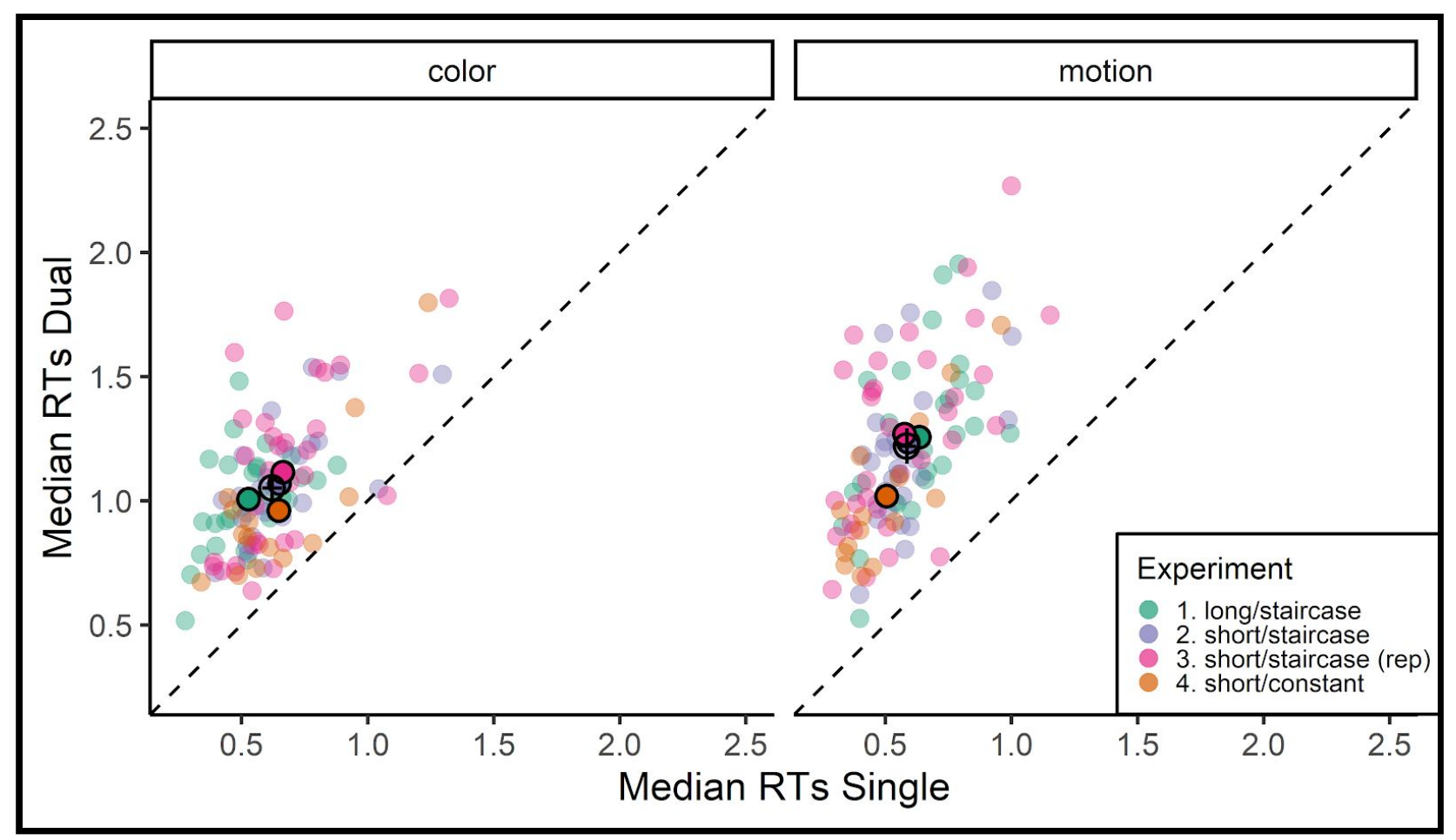

Figure 3 - Response Times

The figure shows participants' median response times in the single- and dual-task conditions, for each task. The dashed diagonal lines show equality in RTs between single and dual conditions. A point above the dashed line means that the participant had slower RTs in the dual- relative to the single-task condition, i.e. they experienced a dual-tasking cost. Every point is one subject in one of the two tasks; colors refer to different experiments (see legend). Group averages for each experiment are shown with the black outlined, color filled shapes. Group averages for each task are shown with the black empty shapes (in this case heavily overlapping with the experiment averages); error bars within these shapes represent the $95 \%$ confidence intervals. For the dual-task condition, we only analysed the RTs for the first of the two tasks on each trial, given that responses were given in a sequential manner.

The median RTs for the Type 1 responses were calculated for each participant and then analysed with linear mixed models in a parallel manner to the one described for discrimination performance. For the dual-task condition, we only analysed the RTs for the first of the two tasks, 
given that responses were done in a sequential manner. Figure 3 shows the median response times for each participant in the two conditions, separately for the two tasks. The best fitting model here had task, condition, and their interactions as fixed effects, a random intercept for each participant and for each experiment. This model intercept $=1.04$, participant $\mathrm{SD}=0.20$, experiment $\mathrm{SD}=0.06$ showed the following effects: a significant effect of condition, so that the dual-task condition predicted longer RTs $\left(\square_{\text {single }}=-0.43,95 \%\right.$ CI $[-0.48,-0.38], \mathrm{t}=17.59, \mathrm{p}<$ $.001)$; an effect of task ( $\square_{\text {motion }}=0.17,95 \%$ CI $\left.[0.12,0.22], \mathrm{t}=6.92, \mathrm{p}<.001\right)$, so that RTs were longer in the motion task; and finally a significant interaction between task and condition, so that this dual-task cost on RTs was more pronounced in the motion task $\left(\square_{\text {motionxsingle }}=-0.20,95 \%\right.$ CI $[-0.27,-0.14], \mathrm{t}=5.96, \mathrm{p}<.001)$. 


\section{Metacognitive Efficiency}

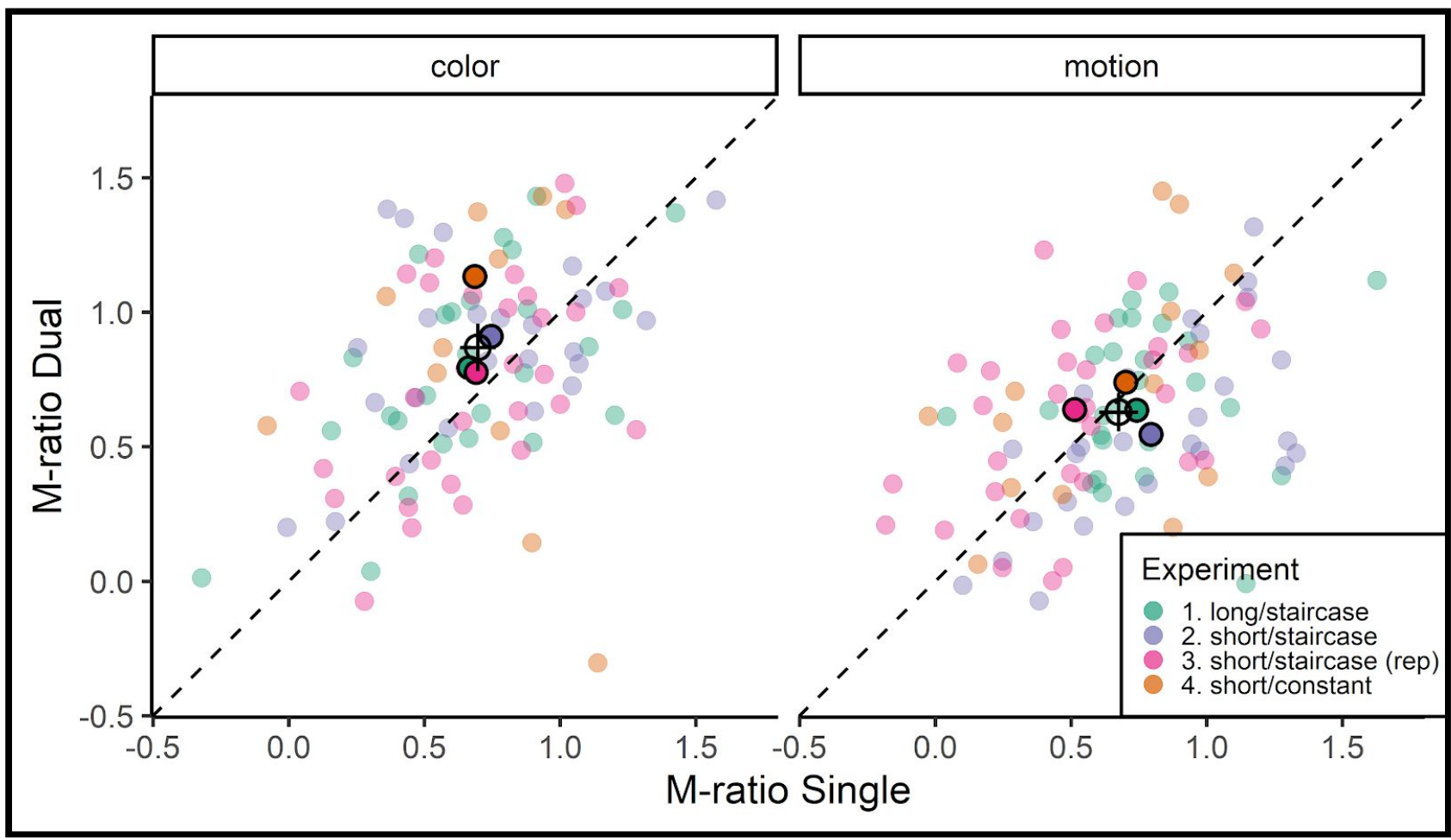

Figure 4 - Metacognitive Efficiency

The figure shows participants' metacognitive efficiency (as measured by the M-ratio) in the single- and dual-trask conditions, in the two tasks. The dashed diagonal lines show equality in metacognitive efficiency in single- and dual-task conditions. A point below the dashed line means that the participant had better metacognitive efficiency in the single than in the dual condition. Every point is one subject in one of the two tasks; colors refer to different experiments. Group averages for each experiment are shown with the black outlined, color filled shapes (the one for the long/staircase experiment is hidden underneath the other ones). Group averages for each task are shown with the black empty shapes; error bars within the empty circles represent the $95 \%$ confidence intervals.

Figure 4 shows the M-ratio measure of metacognitive efficiency for every participant in the two conditions. Here the best fitting model had task, condition, and their interaction as fixed effects, and a random intercept for every participant. This model (intercept $=0.86$, participant $\mathrm{SD}=0.24$ ) had a significant effect of task $\left(\square_{\text {motion }}=-0.24,95 \%\right.$ CI $\left.[-0.32,-0.16], \mathrm{t}=5.63, \mathrm{p}<.001\right)$, 
describing participants' better metacognitive efficiency in the color task; a significant effect of condition $\left(\square_{\text {single }}=-0.17,95 \%\right.$ CI $\left.[-0.25,-0.09], \mathrm{t}=4.00, \mathrm{p}<.001\right)$, showing participants' overall worse metacognition in the single-task condition; and a significant task $\mathrm{X}$ condition interaction $\left(\square_{\text {motionXsingle }}=0.22,95 \%\right.$ CI $\left.[0.10,0.34], \mathrm{t}=3.62, \mathrm{p}<.001\right)$. Post-hoc repeated measure ANOVAs with condition as a within-subject factor were run separately in each task to interpret this interaction, confirming what is shown in Figure 4: participants showed a metacognitive dual-condition benefit in the color task $\left(\mathrm{F}(86)=17.52, \mathrm{p}<.001, \eta^{2}=.044\right.$, partial $\left.\eta^{2}=.226\right)$, while showing no differences in metacognitive efficiency between conditions in the motion task $\left(\mathrm{F}(86)=1.67, \mathrm{p}=.20, \mathrm{BF}_{01}=3.33\right)$.

\section{Control Analyses}

The results from our four experiments were surprising in part. Not only did we not find evidence for a dual-tasking cost on metacognition, but we also observed a small benefit in the task which showed the larger Type 1 cost (the color task). We thus decided to run some control analyses, and an additional experiment, to rule out several potential simple explanations: we explored the possibility that this benefit could be due to an increase in response times (Appendix 1.1) or in participants' motivation in the dual-task condition (Appendix 1.4); in a confidence leak between the two tasks (Appendix 1.2); in a between-conditions difference in stimulus variability (Appendix 1.5); or finally to a strategy (potentially implemented by some participants) of trading off the two tasks (Appendix 1.3). We also analysed if the order of response in the dual-task condition (given our design) was affecting discrimination performance, and found evidence against this hypothesis (Appendix 1.9). Ultimately, none of these potential mechanisms were sufficient to explain our results. 


\section{Discussion}

We adopted a simple visual dual-task paradigm to understand whether the typical dual-task costs in performance associated with multitasking would be accompanied by dual-task costs at the metacognitive level. Across four experiments, we observed a dual-task cost in perceptual discrimination performance and in terms of RTs, but no dual-task cost in metacognitive efficiency. Au contraire, we found evidence for a small, dual-task metacognitive benefit, precisely in the color task. Note that when measuring metacognitive efficiency with the M-diff (a measure closely related to the M-ratio) and metacognitive sensitivity using the area under the Type 2 ROC curve (Appendix 1.8), we found again no dual-task cost on metacognition, suggesting that our results are independent of which specific measure of metacognition is used. We also found no effect of dual-tasking on metacognitive bias (Appendix 1.7), the general tendency of being over- or under-confident, or on the variability of confidence ratings (Appendix 1.10), suggesting that dual-tasking does not affect how individuals anchor or vary their confidence in our setup.

Our study shows that perceptual metacognition can operate efficiently on both tasks in our dual-task condition. This is surprising for two reasons. First, although the information for perceptual decisions is degraded (as can be seen from the dual-task cost at the Type 1 level), it nevertheless remains accessible for a metacognitive judgement in both tasks. In other words, the two metacognitive tasks do not suffer from competing against each other in our paradigm. Second, our results suggest that self-evaluation does not necessarily compete with the central resources that might be needed to coordinate the two concurrent tasks. Further, our results suggest that some information needed for the computation of metacognition (i.e. confidence) is registered early on in the processing stream without degradation caused by the occupation of central processes, and can be read-off and recovered effortlessly when a metacognitive report is required. 
It has previously been shown that confidence can be extracted at an abstract level without loss of information (de Gardelle et al., 2016; de Gardelle \& Mamassian, 2014). Here, we show that perceptual metacognition is resilient in a different way, in that performing two metacognitive tasks instead of one does not appear to degrade metacognition. This result is consistent with a recent study in which participants had to identify two targets within a rapid stream of letters, and had to evaluate their confidence in their reports (Recht et al., 2019). In that study, confidence was not perfectly able to capture the accuracy of reports, but, importantly, in a control experiment metacognitive performance did not depend on whether one or both reports had to be evaluated. Our present result is also in line with the finding of Maniscalco \& Lau (2015) that Type 2 efficiency was unimpaired when a perceptual task was performed concurrently with an easy working memory task.

However, it is important to acknowledge that such resilience of metacognition has its limits. For instance, in the same study, Maniscalco \& Lau (2015) found that Type 2 efficiency could be degraded if the perceptual task was accompanied by a much more demanding working memory task. In a different paradigm, Corallo, Sackur, Dehaene, \& Sigman (2008) had previously shown that participants were introspectively blind to the cost of a secondary concurrent task, when the metacognitive task consisted in the estimation of one's response times. There are thus experimental cases of metacognitive costs induced by multitasking. Nevertheless, these seem to heavily depend on the nature of the interference at the type 1 level, and on the specificity of the metacognitive task.

One intriguing aspect of our results remains the metacognitive benefit found in the dual task condition for the color task. We explored the possibility that this benefit could be due to an increase in response times (Appendix 1.1) or in participants' motivation in the dual-task condition (Appendix 1.4), in a confidence leak between the two tasks (Appendix 1.2), in a between-conditions difference in stimulus variability (Appendix 1.5), or to the strategic forfeiting of one task in favour of the other. None of these potential mechanisms could explain our results. 
Understanding this increase in metacognitive efficiency thus remains an important open issue for future investigations. Notably, our result is consistent with a recent study (Bang et al., 2019) showing that, counterintuitively, increasing sensory noise can result not only in a decrease in Type 1 sensitivity, but also an increase in metacognitive efficiency. Indeed, our dual-task manipulation lowered Type 1 discrimination sensitivity, while metacognitive efficiency increased (in the color task) or was left untouched (in the motion task). As argued by Bang et al. (2019), this pattern is consistent with hierarchical models of metacognition (Fleming \& Daw, 2017; Maniscalco \& Lau, 2016), which allow in principle for dissociations between Type 2 and Type 1 performance like the one we observe in the present study.

But let's go back to our endangered and multitasking pilot from the Introduction. Will they be able to recognise their mistake and safely land their plane? Clearly, there is yet much work to be done to understand the impact of multitasking on metacognition in real-life situations. For once, in our study we asked participants to explicitly rate their confidence in their decisions: although there is evidence that confidence is spontaneously computed when taking a decision (Aguilar-Lleyda et al., 2019; Lebreton et al., 2015), continuously probing someone to evaluate their confidence could help them to maintain awareness on their performance and thus boost their metacognition. One other potentially intriguing factor that could interact in how multitasking affects metacognition, is the level of expertise of the agent: will an expert pilot be more (because of their increased task knowledge), or less (task learning is related to automatization, which is in turn related to decreased conscious awareness) likely to detect their mistake? This is an open question.

The present investigation also used a simple dual-task visual paradigm to measure dual-task costs at the level of perceptual performance and at the metacognitive level. Future experiments could investigate more challenging cases, to further characterize the extent of the resilience of metacognition. For instance, one might use tasks that require exactly the same Type 1 resources (e.g. two independent motion discrimination tasks). Indeed, following current models of 
multitasking interference (Salvucci \& Taatgen, 2008; Wickens, 2002), this situation might give even more leverage to study the factors by which Type 2 multitasking costs relate to Type 1 costs. One other option for further research is to increase the demands put on the cognitive system by engaging the participant in more than two concurrent tasks, so as to reach the multi-tasking limit of the agent. Further, it might be possible to induce a metacognitive cost by use of concurrent working memory (e.g. Maniscalco \& Lau, 2015) and/or sustained attention tasks: indeed, the paradigm used in the present study faced participants with brief trials, requiring focused attention in a limited time frame. Conversely, multitasking situations in real life often involve a continued effort in time. Current work in our lab is in progress to investigate a combination of the situations listed above. 


\section{Acknowledgements}

We would like to thank Jean-Christophe Vergnaud, Pascal Mamassian, David Aguilar Lleyda, Tarryn Baldson, and Samuel Recht for comments during the drafting of this paper. We would also like to thank Audrey Azerot, Maxim Frolov, and Isabelle Brunet for their help in running the experiments presented here. Finally we thank the reviewers of earlier submissions for their insightful comments, which helped to improve this text. This work was made possible by the following fellowships of the Agence Nationale de la Recherche (ANR): for Vincent de Gardelle (ANR-16-CE28-0002; ANR-18-CE28-0015); for Jerome Sackur, Vincent de Gardelle, and Mahiko Konishi (ANR-16-ASTR-0014); for Jerome Sackur and Mahiko Konishi (ANR-17-EURE-0017).

\section{Contributions}

Design: MK, CC, BB, JS, VdG; Data collection: MK, CC; Data Analysis: MK, CC, JS, VdG; Text preparation: MK, JS, VdG. 


\section{References}

Aguilar-Lleyda, D., Konishi, M., Sackur, J., \& de Gardelle, V. (2019). Confidence can be automatically integrated across two visual decisions.

Bang, J. W., Shekhar, M., \& Rahnev, D. (2019). Sensory noise increases metacognitive efficiency. Journal of Experimental Psychology: General, 148(3), 437.

Baranski, J. V., \& Petrusic, W. M. (1998). Probing the locus of confidence judgments: Experiments on the time to determine confidence. Journal of Experimental Psychology: Human Perception and Performance, 24(3), 929.

Bates, D., Mächler, M., Bolker, B., \& Walker, S. (2015). Fitting Linear Mixed-Effects Models Using lme4. Journal of Statistical Software, 67(1), 1-48. https://doi.org/10.18637/jss.v067.i01

Boldt, A., Blundell, C., \& De Martino, B. (2019). Confidence modulates exploration and exploitation in value-based learning. Neuroscience of Consciousness, 2019(1), niz004.

Collette, F., Olivier, L., Van der Linden, M., Laureys, S., Delfiore, G., Luxen, A., \& Salmon, E. (2005). Involvement of both prefrontal and inferior parietal cortex in dual-task performance. Cognitive Brain Research, 24(2), 237-251.

Corallo, G., Sackur, J., Dehaene, S., \& Sigman, M. (2008). Limits on Introspection: Distorted Subjective Time During the Dual-Task Bottleneck. Psychological Science, 19(11), 1110-1117. https://doi.org/10.1111/j.1467-9280.2008.02211.x

Craddock, M. (2018). MetaSDT: Calculate Type 1 and Type 2 Signal Detection Measures. 
https://github.com/craddm/metaSDT

de Gardelle, V., Le Corre, F., \& Mamassian, P. (2016). Confidence as a Common Currency between Vision and Audition. PLOS ONE, 11(1), e0147901.

https://doi.org/10.1371/journal.pone.0147901

de Gardelle, V., \& Mamassian, P. (2014). Does Confidence Use a Common Currency Across Two Visual Tasks? Psychological Science, 25(6), 1286-1288. https://doi.org/10.1177/0956797614528956

Dux, P. E., Ivanoff, J., Asplund, C. L., \& Marois, R. (2006). Isolation of a central bottleneck of information processing with time-resolved fMRI. Neuron, 52(6), 1109-1120.

Finley, J. R., Benjamin, A. S., \& McCarley, J. S. (2014). Metacognition of multitasking: How well do we predict the costs of divided attention? Journal of Experimental Psychology: Applied, 20(2), 158-165. https://doi.org/10.1037/xap0000010

Fleming, S. M., \& Daw, N. D. (2017). Self-evaluation of decision-making: A general Bayesian framework for metacognitive computation. Psychological Review, 124(1), 91-114. https://doi.org/10.1037/rev0000045

Fleming, S. M., \& Dolan, R. J. (2012). The neural basis of metacognitive ability. Phil. Trans. $R$. Soc. B, 367(1594), 1338-1349.

Fleming, S. M., Huijgen, J., \& Dolan, R. J. (2012). Prefrontal contributions to metacognition in perceptual decision making. The Journal of Neuroscience : The Official Journal of the Society for Neuroscience, 32(18), 6117-6125. https://doi.org/10.1523/JNEUROSCI.6489-11.2012

Fleming, S. M., \& Lau, H. C. (2014). How to measure metacognition. Frontiers in Human 
Neuroscience, 8,443 . https://doi.org/10.3389/fnhum.2014.00443

Fleming, S. M., Weil, R. S., Nagy, Z., Dolan, R. J., \& Rees, G. (2010). Relating introspective accuracy to individual differences in brain structure. Science, 329(5998), 1541-1543.

Folke, T., Jacobsen, C., Fleming, S. M., \& De Martino, B. (2017). Explicit representation of confidence informs future value-based decisions. Nature Human Behaviour, 1(1), 0002.

García-Pérez, M. A. (1998). Forced-choice staircases with fixed step sizes: Asymptotic and small-sample properties. Vision Research, 38(12), 1861-1881. https://doi.org/10.1016/S0042-6989(97)00340-4

Guggenmos, M., Wilbertz, G., Hebart, M. N., \& Sterzer, P. (2016). Mesolimbic confidence signals guide perceptual learning in the absence of external feedback. Elife, 5, e13388.

Hainguerlot, M., Vergnaud, J.-C., \& de Gardelle, V. (2018). Metacognitive ability predicts learning cue-stimulus associations in the absence of external feedback. Scientific Reports, 8(1). https://doi.org/10.1038/s41598-018-23936-9

Harvey, N. (1997). Confidence in judgment. Trends in Cognitive Sciences, 1(2), 78-82. https://doi.org/10.1016/S1364-6613(97)01014-0

Henmon, V. A. C. (1911). The relation of the time of a judgment to its accuracy. Psychological Review, 18(3), 186.

Hollard, G., Massoni, S., \& Vergnaud, J.-C. (2016). In search of good probability assessors: An experimental comparison of elicitation rules for confidence judgments. Theory and Decision, 80(3), 363-387.

Hommel, B., Fischer, R., Colzato, L. S., van den Wildenberg, W. P., \& Cellini, C. (2012). The effect of fMRI (noise) on cognitive control. Journal of Experimental Psychology: Human 
Perception and Performance, 38(2), 290.

Horrey, W. J., Lesch, M. F., \& Garabet, A. (2009). Dissociation between driving performance and drivers' subjective estimates of performance and workload in dual-task conditions. Journal of Safety Research, 40(1), 7-12. https://doi.org/10.1016/j.jsr.2008.10.011

Karni, E. (2009). A mechanism for eliciting probabilities. Econometrica, 77(2), 603-606.

Kleiner, M., Brainard, D., Pelli, D., Ingling, A., Murray, R., Broussard, C., \& others. (2007). What's new in Psychtoolbox-3. Perception, 36(14), 1.

Kuznetsova, A., Brockhoff, P. B., \& Christensen, R. H. B. (2017). lmerTest Package: Tests in Linear Mixed Effects Models. Journal of Statistical Software, 82(13), 1-26. https://doi.org/10.18637/jss.v082.i13

Lebreton, M., Abitbol, R., Daunizeau, J., \& Pessiglione, M. (2015). Automatic integration of confidence in the brain valuation signal. Nature Neuroscience, 18(8), 1159.

Linares, D., \& López-Moliner, J. (2016). quickpsy: An R Package to Fit Psychometric Functions for Multiple Groups. The R Journal, 8(1), 122-131.

Lüdecke, D. (2020). sjstats: Statistical Functions for Regression Models (Version 0.17.9). https://doi.org/10.5281/zenodo.1284472

Maniscalco, B., \& Lau, H. (2014). Signal Detection Theory Analysis of Type 1 and Type 2 Data: Meta-d', Response-Specific Meta-d', and the Unequal Variance SDT Model. In S. M. Fleming \& C. D. Frith (Eds.), The Cognitive Neuroscience of Metacognition (pp. 25-66). Springer Berlin Heidelberg. https://doi.org/10.1007/978-3-642-45190-4_3

Maniscalco, B., \& Lau, H. (2015). Manipulation of working memory contents selectively impairs metacognitive sensitivity in a concurrent visual discrimination task. Neuroscience of 
Consciousness, 2015(1). https://doi.org/10.1093/nc/niv002

Maniscalco, B., \& Lau, H. (2016). The signal processing architecture underlying subjective reports of sensory awareness. Neuroscience of Consciousness, 2016(1). https://doi.org/10.1093/nc/niw002

Metcalfe, J. E., \& Shimamura, A. P. (1994). Metacognition: Knowing about knowing. The MIT Press.

Morey, R. D., \& Rouder, J. N. (2018). BayesFactor: Computation of Bayes Factors for Common Designs. https://CRAN.R-project.org/package=BayesFactor

Nelson, T. O., \& Narens, L. (1990). Metamemory: A Theoretical Framework and New Findings. Psychology of Learning and Motivation, 26, 125.

Pashler, H. (1994). Dual-Task Interference in Simple Tasks: Data and Theory. Psychological Bulletin, 116(2), 25.

Peirce, C. S., \& Jastrow, J. (1884). On small differences in sensation.

Prins, N. (2012). The psychometric function: The lapse rate revisited. Journal of Vision, 12(6), $25-25$.

R Core Team. (2018). R: A Language and Environment for Statistical Computing. R Foundation for Statistical Computing. https://www.R-project.org/

Rahnev, D., \& Fleming, S. M. (2019). How experimental procedures influence estimates of metacognitive ability. Neuroscience of Consciousness, 2019(1), niz009.

Rahnev, D., Koizumi, A., McCurdy, L. Y., D’Esposito, M., \& Lau, H. (2015). Confidence Leak in Perceptual Decision Making. Psychological Science, 26(11), 1664-1680. https://doi.org/10.1177/0956797615595037 
Recht, S., Mamassian, P., \& de Gardelle, V. (2019). Temporal attention causes systematic biases in visual confidence. Scientific Reports, 9(1), 1-9.

Salvucci, D. D., \& Taatgen, N. A. (2008). Threaded cognition: An integrated theory of concurrent multitasking. Psychological Review, 115(1), 101-130. https://doi.org/10.1037/0033-295X.115.1.101

Sanbonmatsu, D. M., Strayer, D. L., Biondi, F., Behrends, A. A., \& Moore, S. M. (2016). Cell-phone use diminishes self-awareness of impaired driving. Psychonomic Bulletin \& Review, 23(2), 617-623. https://doi.org/10.3758/s13423-015-0922-4

Sigman, M., \& Dehaene, S. (2005). Parsing a cognitive task: A characterization of the mind's bottleneck. PLoS Biology, 3(2), e37.

Szameitat, A. J., Schubert, T., Müller, K., \& Von Cramon, D. Y. (2002). Localization of executive functions in dual-task performance with fMRI. Journal of Cognitive Neuroscience, 14(8), 1184-1199.

van den Berg, R., Anandalingam, K., Zylberberg, A., Kiani, R., Shadlen, M. N., \& Wolpert, D. M. (2016). A common mechanism underlies changes of mind about decisions and confidence. ELife, 5. https://doi.org/10.7554/eLife.12192

Wichmann, F. A., \& Hill, N. J. (2001). The psychometric function: I. Fitting, sampling, and goodness of fit. Perception \& Psychophysics, 63(8), 1293-1313.

Wickens, C. D. (2002). Multiple resources and performance prediction. Theoretical Issues in Ergonomics Science, 3(2), 159-177. https://doi.org/10.1080/14639220210123806

Wickham, H. (2017). tidyverse: Easily Install and Load the "Tidyverse." https://CRAN.R-project.org/package=tidyverse 
Yu, S., Pleskac, T. J., \& Zeigenfuse, M. D. (2015). Dynamics of postdecisional processing of confidence. Journal of Experimental Psychology: General, 144(2), 489. 


\section{Appendix}

\section{Table of Contents}

1. Supplemental Analyses and Control Experiment 32

1.1 Response Times $\quad 32$

1.2 Confidence Leak 33

1.3 Dual-Task Trade-Off Strategy

1.4 Increased Motivation $\quad 37$

$\begin{array}{ll}1.5 \text { Stimulus Variability } & 38\end{array}$

1.6 Correlations Across Participants 40

1.7 Metacognitive Bias 41

1.8 Area Under the Type 2 ROC Curve and M-diff 42

1.9 Order Effects in Dual-Task Condition 45

1.10 Confidence Ratings 46

2. Linear Mixed Models $\quad 48$

3. Supplementary Plots $\quad 50$ 


\section{Supplemental Analyses and Control Experiment}

\subsection{Response Times}

We set to examine if the difference in response times between the two experimental conditions might explain the metacognitive gain we observe in the color task. Indeed, previous work (Baranski \& Petrusic, 1998) highlighted the close link between RTs and confidence ratings, while other studies (van den Berg et al., 2016; Yu et al., 2015) demonstrated that increasing the RTs of the type 1 or type 2 responses improve metacognitive accuracy. Given that an increase in RTs can lead to improved metacognitive evaluations, and that RTs were longer in our dual-task condition, it is possible that the increase in Type 1 RTs in the dual-task condition was directly linked to the absence of a dual-task Type 2 cost (or even a benefit, as in the case of the color task). In order to test this hypothesis, we looked at the correlation between the dual/single condition difference in RTs and the condition difference in M-ratio for each participant: there was no evidence for such a relation, for either the color task $\left(\mathrm{r}=-0.11, \mathrm{p}=.31, \mathrm{n}=87, \mathrm{BF}_{01}=\right.$ $2.43)$, or the motion task $\left(r=0.04, p=.713, n=87, B_{0}=4.02\right)$. Furthermore, there was no across-participants evidence for a correlation between RTs and M-ratio when looking at the color task (single condition: $\mathrm{r}=0.08, \mathrm{p}=.459, \mathrm{n}=87, \mathrm{BF}_{01}=3.15$; dual condition: $\mathrm{r}=-0.07, \mathrm{p}=$ $.492, \mathrm{n}=87, \mathrm{BF}_{01}=3.33$ ) or the motion task (single condition: $\mathrm{r}=0.16, \mathrm{p}=.14, \mathrm{n}=87, \mathrm{BF}_{01}=$ 1.47; dual condition: $\mathrm{r}=0.07, \mathrm{p}=.539, \mathrm{n}=87, \mathrm{BF}_{01}=3.45$ ). However, the logic of this analysis relies on the assumption that the link between RTs and metacognitive accuracy is constant across participants and experimental conditions, which is not assured in any way. Thus, we believe that we can't rule out completely the possibility that a difference in RTs between conditions contributed to our results. Future work might be able to elucidate this potential mechanism.

\subsection{Confidence Leak}

We investigated the possibility that a within-trial leak of confidence (Rahnev et al., 2015) between tasks helped participants achieve better metacognition in the dual condition. Confidence leak refers to the observed effect that confidence ratings are influenced by inter-trial and 
inter-task previous confidence ratings, and this has been shown to be an effect independent from simple attentional fluctuations or response priming. While such confidence leak can be detrimental to metacognition if every trial or task were completely independent from each other (see negative correlations of confidence leak with M-ratio in Experiments 3 and 4 in Rahnev et al., 2015), in three out of our four experiments we used a staircase procedure that started from a very easy stimulus level for both tasks: this inevitably leads to a certain level of dependency between the two tasks, and does open up the possibility that a confidence leak might be beneficial to metacognition in the dual-task condition. We first confirmed that participants exhibited an inter-task confidence leak in our dual-task condition : we constructed linear mixed models with participants as random factors, describing the second (in order of response) task's confidence ratings on each trial depending on the discrimination accuracy for that task (correct/mistake), the stimulus strength of the second task, and the confidence rating given for the first task in that trial. We then compared models with and without the first confidence rating through a likelihood ratio test, which confirmed that a model containing the first confidence rating was significantly better than one with just stimulus strength and accuracy in describing the second confidence ratings $(\chi 2(1)=218.29, \mathrm{p}<.001)$. Next, we decided to quantify the amount of confidence leak that each participant exhibited, to see if it would correlate with their M-ratio in the dual-task condition (and specifically in the color task, which showed the dual-task Type 2 benefit). To do this, we looked at the average confidence ratings given by each participant in the color task (and only when the color task was the second response in that trial), depending on the accuracy of the response to the color task and of the motion task in the same trial, resulting in four average confidence levels per participant, i.e. confidence given for the response in the color task when both mistakes were done in both tasks in the same trial; when both tasks were correct; color-correct/motion-wrong; color-wrong/motion-correct. Next, we computed two values per participant of the leak they experienced, by subtracting the confidence of color-correct/motion wrong from the color-correct/motion-correct value, and subtracting the confidence of color-wrong/motion-wrong from the color-wrong/motion-correct value (we removed two participants from these analyses as they were never concurrently wrong in both tasks on the same trial): for each of these values, the accuracy of the color task does not vary in the two measures 
that are subtracted, so if participants are not influenced by their first confidence rating, these values should be at zero. That is, if the second confidence rating is truly independent from the

first one, the average confidence given to a correct response in the color task should not change depending on the correctness of the response in the motion task. Finally, we averaged these values, obtaining one measure of confidence leak in the color task for each participant. A one-sample t-test confirmed that most participants experienced a small positive confidence leak $($ mean leak $=1.59 \%$ on the confidence scale; $t(87)=2.86, p=.005)$. However, this leak was not correlated with metacognitive efficiency in the dual condition of the color task $(\mathrm{r}=-0.15, \mathrm{p}=$ $.165, \mathrm{n}=87$ ). A Bayes factor for this correlation provided no strong evidence for either hypothesis $\left(\mathrm{BF}_{01}=1.51\right)$. Thus, we did not find evidence to show that a confidence leak helped participants achieve better metacognition in the dual condition, although we don't have strong evidence to rule out this possibility completely.

\subsection{Dual-Task Trade-Off Strategy}

Here, the possibility that we investigated is that in the dual-condition, participants might adopt a trade-off strategy. For example, and contrary to instructions, participants might have performed the two tasks serially and first performed the motion task, before attending to the color task if time allowed. In terms of performance, this could induce a strong dual-task cost for the color task but not for the motion task. Further, participants would know whether they did attend to the color task or not, which could improve their metacognitive efficiency. This pattern could have also emerged if the motion task automatically attracted all of participants' attention, and if participants noticed it. Interestingly, if this lack of attention occurred on some but not all trials, this would produce two effects for the color task: a lower type 1 performance but also a better correlation between performance and confidence across trials (and therefore a higher M-ratio), which is what our data showed. To assess this possible scenario, we reasoned that if different participants engage differently in this strategy, we would expect a correlation across-participants between the dual-task type 2 benefit and the dual-task type 1 cost measured in the color task. 
Firstly, we constructed linear mixed models where M-ratio values were predicted by discrimination sensitivity, task type and task condition as fixed effects, and participants and experiment as random effects. The best fitting model had an interaction of all three fixed effects. To better understand this triple interaction, we looked at the correlation between discrimination sensitivity and M-ratio in the different conditions. In the dual condition of the color task: this correlation was negative $(\mathrm{r}=-0.23, \mathrm{p}=.032, \mathrm{n}=87)$, which was not present for the motion task $\left(\mathrm{r}=0.03, \mathrm{p}=.782, \mathrm{n}=87, \mathrm{BF}_{01}=4.32\right)$ or for the single condition in either color $(\mathrm{r}=-0.08, \mathrm{p}=$ $\left..461, \mathrm{n}=87, \mathrm{BF}_{01}=3.51\right)$ or motion $\left(\mathrm{r}=0.1, \mathrm{p}=.356, \mathrm{n}=87, \mathrm{BF}_{01}=3.06\right)$.

Secondly, we looked at the relationship between the dual-task cost in discrimination sensitivity (sensitivity in dual minus sensitivity in single condition), and the M-ratio differences in conditions (M-ratio in dual minus M-ratio in single condition) for every participant. Again, there was a significant negative correlation $(\mathrm{r}=-0.22, \mathrm{p}=.04, \mathrm{n}=87)$ in the color task, which was not present in the motion task $\left(\mathrm{r}=-0.15, \mathrm{p}=.165, \mathrm{n}=87, \mathrm{BF}_{0_{1}}=1.89\right)$ : that is, when performing the color task, participants who suffered a greater cost in Type 1 discrimination sensitivity also exhibited a greater dual-task benefit in metacognitive efficiency.

Finally, to understand if participants actually adopted a strategy of selectively sacrificing the color task, we fitted psychometric curves for every participant, for the motion task in the dual-task condition, depending on the accuracy of the color task in the same trial: the logic that we used here is that, if participants indeed adopt such a strategy, they should exhibit better discrimination sensitivity in the motion task when they made a mistake in the same trial in the color task. Inspection of the scatterplot of motion sensitivity when same-trial response for color was correct versus when color was incorrect (see Figure B), revealed three clearly outlying subjects, for which motion discrimination sensitivity was much higher when making mistakes in the color task: of these three subjects, two showed a dual-task benefit of M-ratio in the color task, while one showed a dual-task cost. Nevertheless, the overall trend did not show this tendency: a paired-sample t-test revealed no significant difference between motion discrimination sensitivity for color-correct and color-error conditions even including the outlying subjects $\left(\mathrm{t}(86)=1.54, \mathrm{p}=.128, \mathrm{BF}_{0_{1}}=2.65\right)$, although the evidence in favour of the null was 
much stronger when removing them $\left(\mathrm{t}(83)=0.02, \mathrm{p}=.982, \mathrm{BF}_{0_{1}}=8.11\right)$. Lastly, we checked if this difference in motion discrimination sensitivity (between color-correct and color-error conditions) correlated with the M-ratio benefit for the color task in the dual-task condition: there was no sign of such a relation $(\mathrm{r}=0.02, \mathrm{p}=.85, \mathrm{n}=87)$.

In conclusion, we believe that participants did not consistently adopt this strategy for two reasons. Firstly, there's no evidence that participants sacrificed one task for the benefit of the other. Secondly, in this hypothesis participants would always perform the motion task first, and then the color task if time allowed. If such time pressure was determinant for our results, the results from our experiment using a $1500 \mathrm{~ms}$ stimulus duration would tend to diverge from the ones of the other experiments using a 300ms duration. This was not the case.

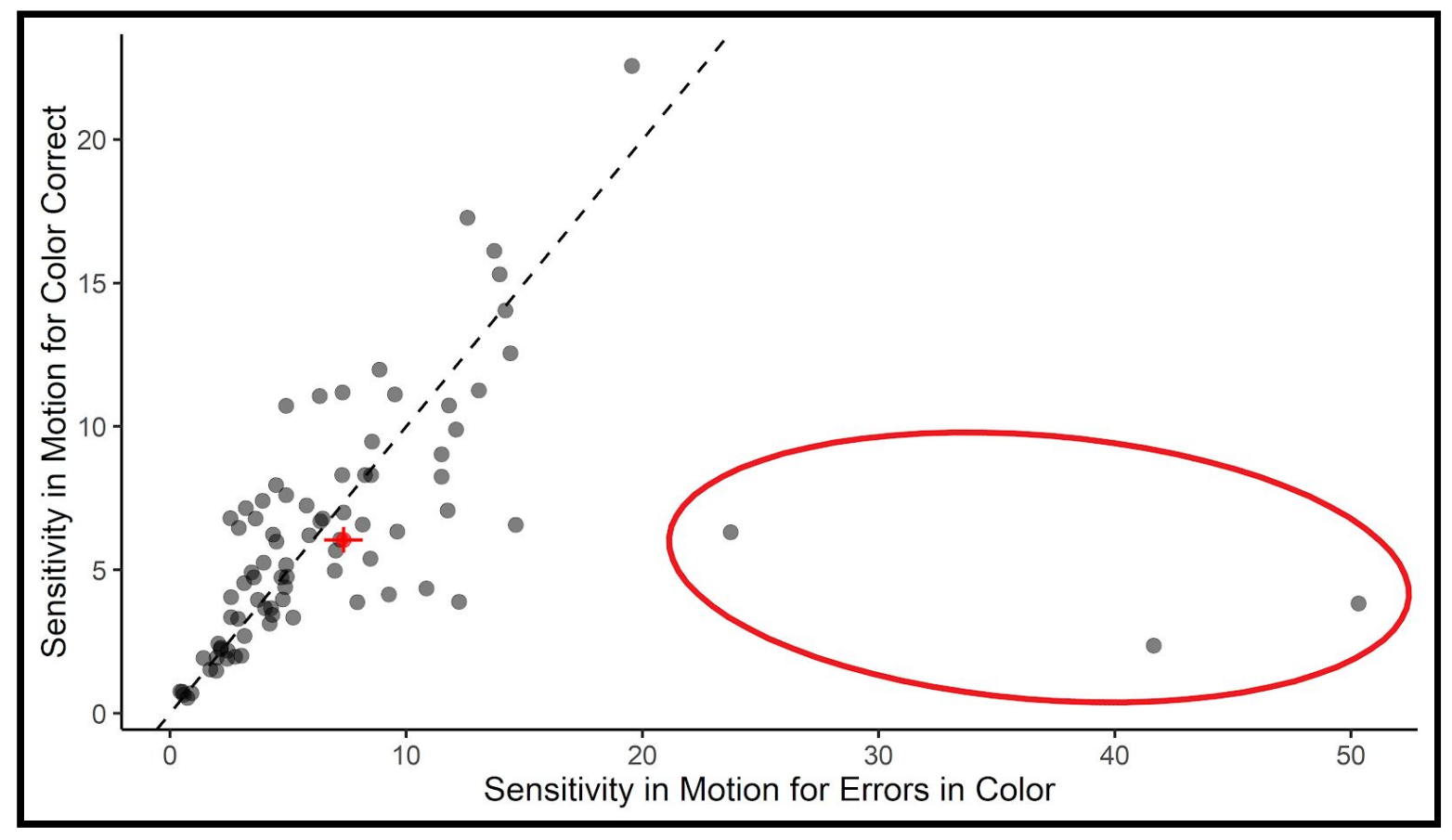

Figure B - Motion Discrimination Dependent on Color Accuracy

Scatterplot of participants' sensitivity in the dual condition of the motion discrimination task, dependent on the accuracy in the color task, on the same trial. While most participants don't show any bias, three participants (highlighted in red) clearly show a 
sharp increase in motion discrimination sensitivity when they made an incorrect response in the color task, suggesting that they might've intentionally sacrificed the color task on several trials in order to focus exclusively on the motion task. Group average is displayed with the red dot. Error bars represent the standard error of the mean.

\subsection{Increased Motivation}

Finally, we investigated whether participants' metacognitive abilities could have been boosted by an increase in motivation in the dual-task condition, which is more challenging (e.g. Hommel et al., 2012). We ran an additional control experiment at the Laboratory of Experimental Economics in Paris (LEEP) that used the same parameters as the short duration / staircase experiment, but that also modulated participants' motivation by varying the reward structure: each 30 trial block was divided in two 15-trial blocks, one of which used the same pay-off structure of the short/staircase and long/staircase experiments ran at the LEEP (detailed in the Methods/Participants section), while the other paid off twice as much. The order of these blocks was randomised, and participants were told which block was coming up beforehand. Thirty-three participants took part in the study, and three were excluded from analysis following our exclusion criteria, leaving thirty participants for all analyses concerning this experiment.

Critically, we ran a repeated-measures ANOVA with M-ratio as the dependant variable, and task (color/motion), condition (single/dual), motivation (high/normal), and their interactions, as fixed factors: none of the factors or interactions, and importantly the motivation factor $(\mathrm{F}(29)=0.328$, $\mathrm{p}=.571$ ), were significant. In this case we also used the BayesFactor (Morey \& Rouder, 2018) package in $\mathrm{R}$ to compare models with and without the motivation factor and its interactions with other factors: the model without motivation was preferred by a factor of 95, while a model predicting M-ratio with only motivation as a fixed effect and a random intercept for each participant had $\mathrm{a} \mathrm{BF}_{0}$ of 5.92. We also confirmed the dual-task costs on Type 1 discrimination sensitivity: a rm-ANOVA describing discrimination sensitivity with task and condition as factors revealed: a significant effect of task (lower sensitivity in the motion task; $F(29)=110.9, p<$ .001 ); a significant effect of condition (lower sensitivity in the dual-task condition; $F(29)=$ $30.65, \mathrm{p}<.001)$; and a significant task $\mathrm{X}$ condition interaction $(\mathrm{F}(29)=29.72, \mathrm{p}<.001)$. In sum, our reward modulation had no impact on metacognitive efficiency. 


\subsection{Stimulus Variability}

A recent study (Rahnev \& Fleming, 2019) showed that, by exposing observers to different levels of a stimulus, staircase methods produce inflated measures of metacognition when compared to constant stimuli methodologies. As we used separate staircases in our two experimental conditions, if stimulus variability was to be higher in the dual-task condition, this could potentially inflate participants' metacognitive efficiency compared to the single-task condition, thus providing a simple explanation for our result. In order to examine this possibility, we ran a repeated measures anova describing the effects of task and condition on the standard deviation (SD) of the stimulus levels (dots' motion coherence for the motion task, and proportion of red VS blue dots in the color task) that each participant was exposed to in the experiments. Neither the condition factor $\left(\mathrm{F}(1,86)=2.41, \mathrm{p}=.12, \mathrm{BF}_{01}=3.47\right)$, or the interaction between task and condition $(\mathrm{F}(1,86)=0.82, \mathrm{p}=.36$; the model without an interaction was preferred by a Bayes factor of 4.9) were significant, suggesting that stimulus variability was comparable in our two experimental conditions. We also repeated this analysis by using the variability in the subjective stimulus values, that are, the physical value of each stimulus converted into a signal-to-noise ratio, given the psychometric curve for each participant in each condition. In this case, the rm-ANOVA provided evidence that stimulus variability was higher in the single-task condition $(\mathrm{F}(1,86)=30.69, \mathrm{p}<.001)$, as well as higher in the motion task overall $(\mathrm{F}(1,86)=22.64, \mathrm{p}<$ $.001)$; the interaction between task and condition was not significant $(F(1,86)=1.73, p=.19)$, and the model without an interaction term was prefered by a Bayes factor of 3.6. In sum, stimulus variability was not higher in the dual-task condition, suggesting that this potential mechanism cannot account for our results. Figure C shows the SD of subjective stimulus levels presented to participants in the four experiments, by task and condition. 


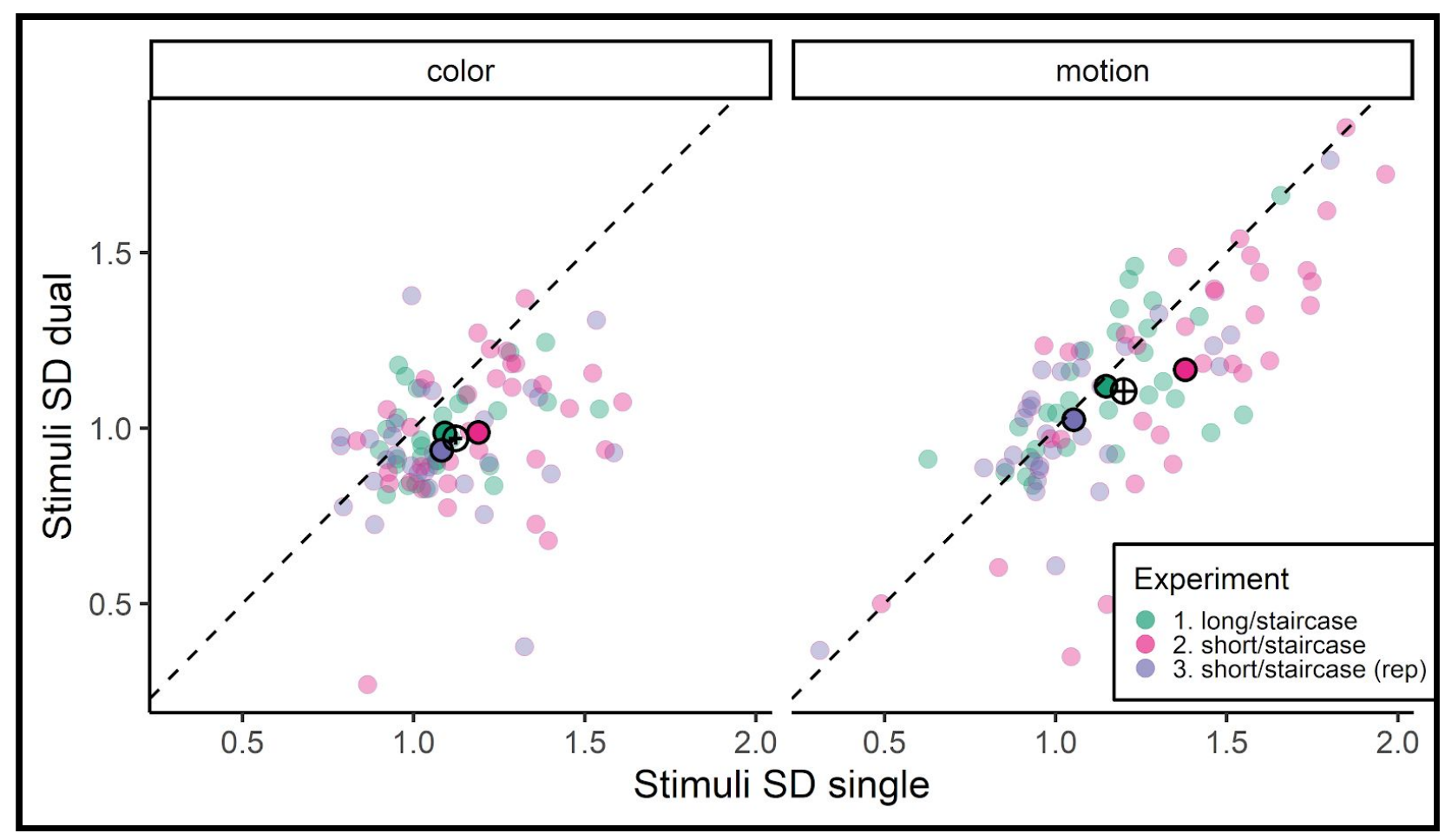

Figure C - Stimulus Variability

The figure shows the standard deviation of subjective stimulus variability as experienced by participants in the three staircase experiments, for each task and condition separately. The dashed diagonal lines show equality in stimulus variability between conditions. A point above the dashed line means that the participant had higher stimulus SD in the dual- relative to the single-task condition, i.e. they experienced more variable stimuli in the dual-task condition. Every point is one subject in one of the two tasks; colors refer to different experiments (see legend). Group averages for each experiment are shown with the black outlined, color filled shapes. Group averages for each task are shown with the black empty shapes (in this case heavily overlapping with the experiment averages); error bars within these shapes represent the standard error of the mean. The constant stimuli experiment has zero variability, as only one level of stimulus was presented to each participant throughout the experiment.

\subsection{Correlations Across Participants}

Metacognitive efficiency (M-ratio) was significantly correlated across participants between the color and the motion task for both the single-task condition $(\mathrm{r}=0.35, \mathrm{p}=.001, \mathrm{n}=87)$ and the dual-task condition $(\mathrm{r}=0.51, \mathrm{p}<.001, \mathrm{n}=87)$. It was also significantly correlated between the single-task and dual-task condition, for both the color task $(\mathrm{r}=0.52, \mathrm{p}<.001, \mathrm{n}=87)$ and the 
motion task $(\mathrm{r}=0.48, \mathrm{p}<.001, \mathrm{n}=87)$. Additionally, between-task metacognitive bias was highly correlated both in the single-task condition $(\mathrm{r}=0.76, \mathrm{p}<.001, \mathrm{n}=87)$ and in the dual-task condition $(\mathrm{r}=0.79, \mathrm{p}<.001, \mathrm{n}=87)$. Bias was also highly correlated within-task, across conditions, for both the color task $(\mathrm{r}=0.85, \mathrm{p}<.001, \mathrm{n}=87)$ and the motion task $(\mathrm{r}=0.79, \mathrm{p}<$ $.001, \mathrm{n}=87)$.

\subsection{Metacognitive Bias}

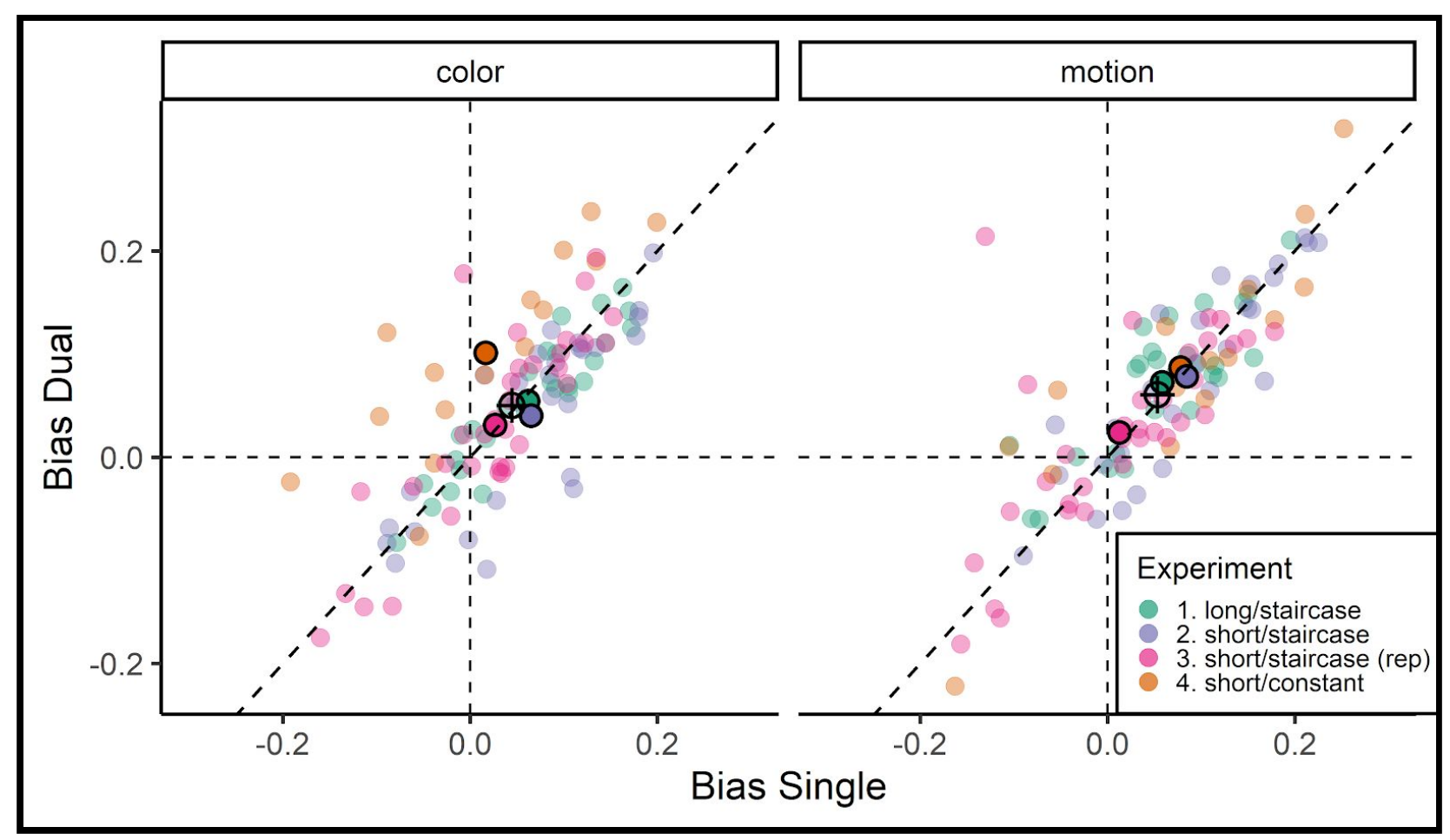

Figure D - Metacognitive Bias

The figure shows the metacognitive bias as average confidence minus average accuracy in units 0 to 1 , for each task and condition separately. E.g. a participant with $82 \%$ average confidence and $78 \%$ accuracy in one task and one condition, would have a positive overconfidence bias of $0.82-0.78=0.04$. The dashed vertical line shows the point of no bias for the single condition; the dashed horizontal line shows the same for the dual condition; the dashed diagonal line shows the point of no difference in bias between single and dual conditions. Every point is one subject in one of the two tasks; colors refer to different experiments (see legend). Group averages for each task are shown with the black empty shapes (again overlapping with experiment averages); error bars within the shapes show the $95 \%$ confidence intervals. 
Metacognitive bias, also known as over/under-confidence or calibration, is a difference in subjective confidence despite basic task performance remaining constant (Fleming \& Lau, 2014). Previous research (Horrey et al., 2009; Sanbonmatsu et al., 2016) had found a general pattern of over-confidence when multitasking; this is in line with the finding that people are usually overconfident, and especially so when involved in a difficult task (Harvey, 1997). Following this, we also expected our participants to show an over-confidence bias in the dual- relative to the single-task condition.

Bias was thus computed for each participant, in each task and condition separately, as their average confidence level (from 50 to 100) minus their average Type 1 performance accuracy (from 50 to $100 \%$ ). This means that a positive bias signified over-confidence while a negative one would signal under-confidence. We then tested the effects of condition and task with linear mixed models, in a parallel fashion as for metacognitive efficiency. A list of the best models tested is available in the next subsection, together with their degrees of freedom and goodness-of-fit statistics relative to each other. Figure D shows the metacognitive bias that participants showed in the two tasks and in the two conditions. The best fitting model for this relation was a simple model that had no fixed effects, a random slope and intercept for the effect of task and condition for every subject, and a random intercept and slope for the effect of task for each experiment. Indeed, as suggested graphically, there was no evidence that participants were differently biased between tasks or conditions, but instead displayed a general over-confidence bias: this was confirmed by a one-sample t-test on the whole dataset (mean bias $=0.05 ; \mathrm{t}(86)=$ $6.53, \mathrm{p}<.001$, Cohen's $d=0.7)$. The average bias in the color task, in the constant stimuli experiment, appears to diverge with the overall pattern of results in Figure D: however, the staircase/constant stimuli factor was included as a fixed effect but rejected from every model using backwards elimination with Satterthwaite method (as described in the Methods section). In conclusion, we do not find a dual-task effect on participants' metacognitive bias: observers were generally overconfident, but equally so when doing one, or two tasks at the same time. 


\subsection{Area Under the Type 2 ROC Curve and M-diff}

In the field of metacognition it is still debated which measures of metacognitive sensitivity and efficiency better capture this cognitive process. We thus decided to replicate our main analyses by using two alternative measures: M-diff (a measure closely related to the M-ratio) for metacognitive efficiency, and the area under the type 2 ROC curve (AUROC2) for metacognitive sensitivity. We analysed the effects of task and condition describing the M-diff for each participant, with the same best fitting LMM used for the M-ratio: this model had task, condition, and their interaction as fixed effects, and a random intercept for each participant. The results mirrored those for the M-ratio: there was a significant effect of task $(t=5.05, p<.001)$, describing participants' better metacognitive efficiency in the color task; a significant effect of condition $(\mathrm{t}=4.18, \mathrm{p}<.001)$, showing participants' overall worse metacognition in the single-task condition; and a significant task $\mathrm{X}$ condition interaction $(\mathrm{t}=3.18, \mathrm{p}=.002)$. Post-hoc repeated measure ANOVAs with condition as a within-subject factor were run separately in each task to interpret this interaction: again, participants showed a metacognitive dual-condition benefit in the color task $\left(\mathrm{F}(1,86)=21.81, \mathrm{p}<.001, \eta^{2}=.052\right.$, partial $\left.\eta^{2}=.319\right)$, while showing no differences in metacognitive efficiency between conditions in the motion task $(\mathrm{F}(1,86)=0.12$, $\left.\mathrm{p}=.732, \mathrm{BF}_{01}=6.36\right)$.

We also analysed the effects of task and condition using the area under the type 2 ROC curve (AUROC2) as a measure of metacognitive sensitivity (Fleming \& Lau, 2014; Galvin et al., 2003). The Type 2 ROC curve is another popular way to measure metacognition, but, unlike the M-ratio, is known to be dependent on Type 1 performance. This means that a decrease in AUROC2 would be expected, and not surprising, given a dual-task cost decrease in Type 1 performance. Instead, mirroring what found for the M-ratio, we found no cost of dual-tasking, on Type 2 performance: the condition effect on AUROC2 was tested with within-subject ANOVAs separately for the color task $\left(\mathrm{F}(86)=0.106, \mathrm{p}=.745, \mathrm{BF}_{01}=6.29\right)$, and motion task $(\mathrm{F}(86)=$ $\left.3.29, \mathrm{p}=.07, \mathrm{BF}_{0_{1}}=1.78\right)$. Figure $\mathrm{E}$ shows the AUROC2 for each participant, in the two experimental conditions, for each task. 


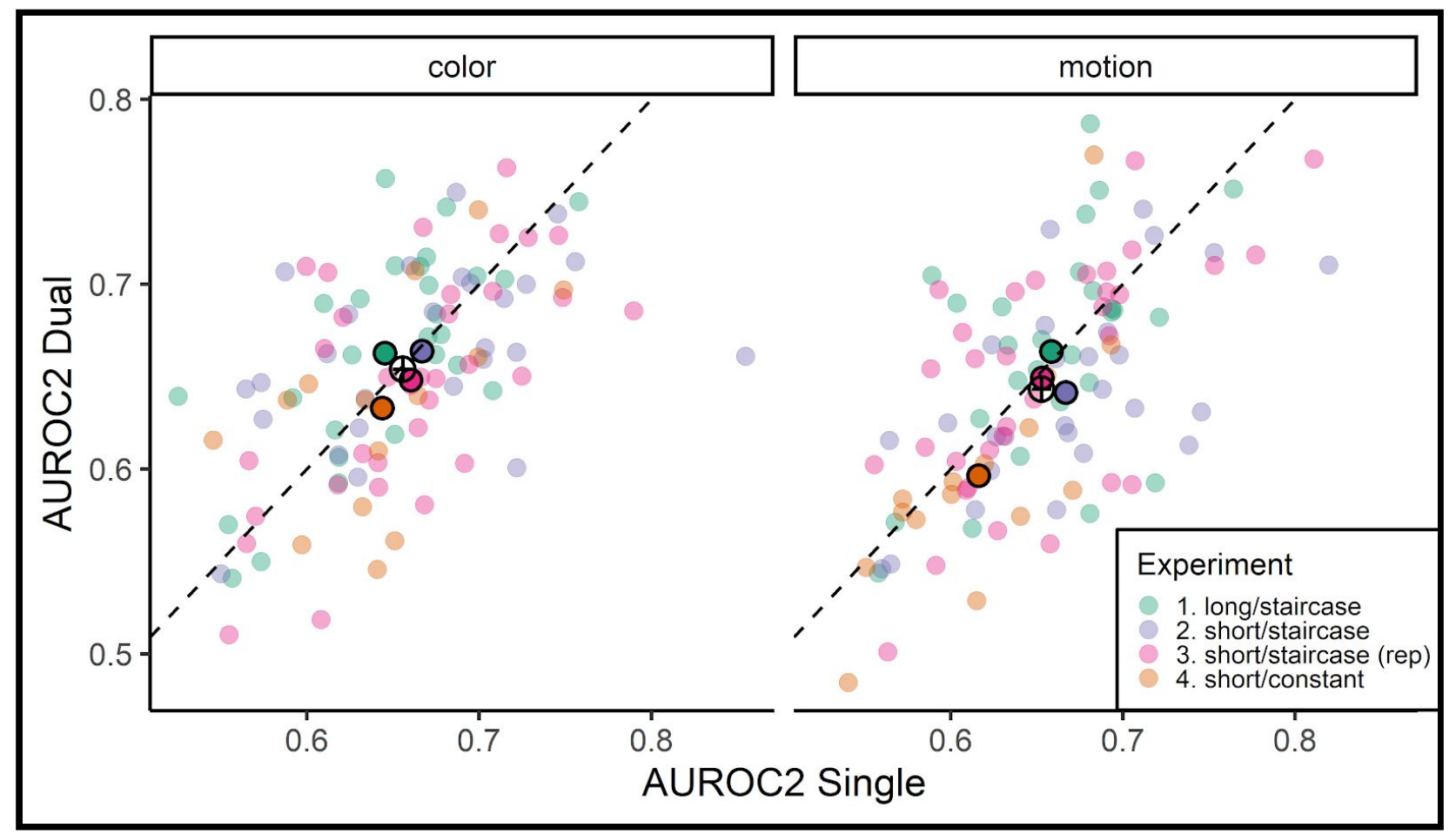

Figure E - Metacognitive Sensitivity - AUROC2

The figure shows the area under the Type 2 curve (AUROC2) for each participant, in each task, for the two experimental conditions. The dashed diagonal lines show equality in AUROC2 between single and dual conditions. A point below the dashed line means that the participant had increased AUROC2 in the single- relative to the dual-task condition, i.e. they experienced a dual-tasking cost. Every point is one subject in one of the two tasks; colors refer to different experiments (see legend). Group averages for each experiment are shown with the black outlined, color filled shapes. Group averages for each task are shown with the black empty shapes (in this case heavily overlapping with the experiment averages); error bars within these shapes represent the standard error of the mean. 


\subsection{Order Effects in Dual-Task Condition}

Our four experiments (plus the motivation control experiment) all followed the same design, in which participants in the dual-task condition gave a discrimination response plus a confidence judgment for one task (e.g. the color task), and then the same two responses for the other task (e.g. the motion task). This left open the possibility that some order effects could be in play: for example, that only the task to which participants responded second would incur in a discrimination or a metacognitive cost (e.g. because of memory decay effects), or vice versa.

In order to control for such order effects for discrimination performance, we fit psychometric curves for each participant in a similar fashion described in the Methods section: this time we took only dual-task trials, and looked at each task with order of response as our grouping factor. We then analysed the effect of order of response on the slope of such psychometric fitted curves with a rm-ANOVA. For the color task, the ANOVA provided no evidence of such effects, with the Bayes factor providing moderate evidence for no effect of response order on discrimination performance $\left(\mathrm{F}(1,86)=1.42, \mathrm{p}=.237, \mathrm{BF}_{0_{1}}=4.15\right)$. Analogous results were found in the motion task $\left(\mathrm{F}(1,86)=1.55, \mathrm{p}=.215, \mathrm{BF}_{0_{1}}=4.80\right)$.

Further, we set to replicate our LMM analyses on discrimination performance, this time separately for first-responded, and second-responded tasks, to see if there would be any differences. We described the same LMM of our main analyses of discrimination performance, with task, condition, stimulus duration, and their interactions as fixed effects, a random intercept for each participant, and a random intercept by experiment: the step function again suggested this

was the best fitting model. In both first, and second responded tasks the models suggested nearly identical results. For first-responded tasks, the model (intercept $=10.03$, participant $\mathrm{SD}=1.83$, experiment $\mathrm{SD}=0.54$ ) had a significant effect of condition, so that the dual-task condition predicted lower discrimination sensitivity $\left(\square_{\text {single }}=9.55,95 \%\right.$ CI $\left.[7.51], \mathrm{t}=11.61, \mathrm{p}<.001\right)$; a significant effect of stimulus duration $\left(\square_{\text {short }}=-4.31,95 \% \mathrm{CI}[-6.14,-2.36], \mathrm{t}=3.85, \mathrm{p}=.025\right)$ so that the longer duration predicted higher sensitivity; a significant interaction between task and 
condition, to the effect that the dual-task cost on sensitivity was increased in the color task $\left(\square_{\text {motionXsingle }}=-6.76,95 \%\right.$ CI $\left.[-9.62,-3.90], \mathrm{t}=4.61, \mathrm{p}<.001\right)$. For second-responded tasks, again the model (intercept $=9.19$, participant $\mathrm{SD}=2.12$, experiment $\mathrm{SD}=0.82$ ) had a significant effect of condition, so that the dual-task condition predicted lower discrimination sensitivity $(\square$ single $=10.10,95 \%$ CI $[8.09,12.16], \mathrm{t}=9.71, \mathrm{p}<.001)$; a near-significant effect of stimulus duration $\left(\square_{\text {short }}=-4.20,95 \%\right.$ CI $\left.[-5.78,-2.10], \mathrm{t}=3.14, \mathrm{p}=.055\right)$ so that the longer duration predicted higher sensitivity; and finally a significant interaction between task and condition, to the effect that the dual-task cost on sensitivity was increased in the color task $\left(\square_{\text {motionXsingle }}=\right.$ $-7.07,95 \%$ CI $[-9.89,-4.25], \mathrm{t}=4.89, \mathrm{p}<.001)$.

In summary, we don't find evidence for order effects on discrimination performance (and instead find some evidence for the null hypothesis of no order effects) with our experimental design.

\subsection{Confidence Ratings}

We analysed the effects of response accuracy (correct/incorrect), condition (single/dual), and task (color/motion) on the raw confidence ratings of each participant, with a rm-ANOVA. This revealed the following effects: an effect of accuracy with higher confidence ratings for correct responses $\left(\mathrm{F}(1,86)=468.8, \mathrm{p}<.001, \eta^{2}=.217\right.$, partial $\left.\eta^{2}=.919\right)$; an effect of condition with higher confidence in the single-task condition $\left(\mathrm{F}(1,86)=10.4, \mathrm{p}=.002, \eta^{2}=.004\right.$, partial $\eta^{2}=$ $.162)$; an effect of task with higher confidence for the motion task $\left(F(1,86)=8.05, p=.006, \eta^{2}=\right.$ .004 , partial $\eta^{2}=.169$ ); a condition $\mathrm{X}$ task interaction with a larger condition difference in terms of confidence ratings in the color task $\left(F(1,86)=11.32, p=.001, \eta^{2}=.002\right.$, partial $\left.\eta^{2}=.100\right)$; and finally a triple interaction $\left(\mathrm{F}(1,86)=5.17, \mathrm{p}=.025, \eta^{2}=.000\right.$, partial $\left.\eta^{2}=.017\right)$. 
We also analysed the variability of confidence ratings (as the SD of confidence ratings of each participant) in analogous fashion. This time the rm-ANOVA had a significant effect of task $\left(F(1,86)=9.83, p=.002, \eta^{2}=.005\right.$, partial $\left.\eta^{2}=.132\right) ;$ an effect of accuracy $(F(1,86)=31.09, p$ $<.001, \eta^{2}=.046$, partial $\left.\eta^{2}=.578\right)$; a condition $X$ task interaction $\left(F(1,86)=5.74, p=.018, \eta^{2}=\right.$ .002 , partial $\left.\eta^{2}=.051\right)$; a condition $X$ accuracy interaction $\left(F(1,86)=6.79, p=.011, \eta^{2}=.002\right.$, partial $\left.\eta^{2}=.069\right)$; a task $X$ accuracy interaction $\left(\mathrm{F}(1,86)=14.5, \mathrm{p}<.001, \eta^{2}=.006\right.$, partial $\eta^{2}=$ $.153)$; and a triple interaction $\left(\mathrm{F}(1,86)=14.1, \mathrm{p}<.001, \eta^{2}=.003\right.$, partial $\left.\eta^{2}=.088\right)$. Interestingly, there was evidence against a main effect of condition $(\mathrm{F}(1,86)=0.99, \mathrm{p}=.322$, $\left.\mathrm{BF}_{0_{1}}=10.81\right)$, suggesting that the experimental condition did not influence the variability of participants' confidence ratings. These results are summarised in Table X below.

\begin{tabular}{|c|c|c|c|c|}
\hline Task & Condition & Accuracy & Mean Confidence & SD Confidence \\
\hline Color & Single & Correct & 85.9 & 7.8 \\
\hline Color & Single & Incorrect & 77.0 & 8.9 \\
\hline Color & Dual & Correct & 84.1 & 7.8 \\
\hline Color & Dual & Incorrect & 74.5 & 10.2 \\
\hline Motion & Single & Correct & 86.8 & 7.9 \\
\hline Motion & Single & Incorrect & 76.9 & 9.7 \\
\hline Motion & Dual & Correct & 85.9 & 8.1 \\
\hline Motion & Dual & Incorrect & 77.0 & 9.8 \\
\hline
\end{tabular}

Table X 


\section{Linear Mixed Models}

\begin{tabular}{|c|c|c|c|c|c|}
\hline Analysis & Model & Df & $\mathbf{A I C}$ & BIC & Deviance \\
\hline $\begin{array}{l}\text { Discrimination } \\
\text { performance }\end{array}$ & $\begin{array}{c}\text { Discrimination sensitivity } \sim \text { task } \\
* \text { condition*stimDuration }+(1 \mid \text { subject })+ \\
(1 \mid \text { experiment })\end{array}$ & 11 & 2487.9 & 2532.0 & 2465.9 \\
\hline $\begin{array}{l}\text { Metacognitive } \\
\text { efficiency }\end{array}$ & M-ratio $\sim$ task $*$ condition $+(1 \mid$ subject $)$ & 6 & 2399.0 & 2424.2 & 2387.0 \\
\hline $\begin{array}{l}\text { Response } \\
\text { times }\end{array}$ & $\begin{array}{c}\mathrm{RTs} \sim \operatorname{task} * \text { condition }+(1 \mid \text { subject })+ \\
(1 \mid \text { experiment })\end{array}$ & 7 & -86.44 & -58.36 & -100.44 \\
\hline $\begin{array}{l}\text { Metacognitive } \\
\text { bias }\end{array}$ & $\begin{array}{c}\text { Bias } \sim 1+\underset{(\text { task }+ \text { condition } \mid \text { subject })}{ }+ \\
(\text { task } \mid \exp )\end{array}$ & 11 & -1132.9 & -1092.8 & -1152.9 \\
\hline
\end{tabular}

Table Y

In order to be able to estimate the size of the dual-tasking effect on discrimination performance and on metacognitive efficiency, we replicated the analyses reported in the Results section this time standardising the dependent variable of each model.

The model for discrimination performance had task, condition, stimulus duration, and their interactions as fixed effects, a random intercept for each participant, and a random intercept by experiment. This model (intercept $=0.56$, participant $\mathrm{SD}=0.36$, experiment $\mathrm{SD}=0.21$ ) revealed the following effects of interest: a significant effect of condition, so that the dual-task condition predicted lower discrimination sensitivity $\left(\square_{\text {single }}=1.16,95 \%\right.$ CI $\left.[0.76,1.56], \mathrm{t}=5.73, \mathrm{p}<.001\right)$; a significant effect of stimulus duration $\left(\square_{\text {short }}=-1.00,95 \%\right.$ CI $\left.[-1.54,-0.47], \mathrm{t}=3.29, \mathrm{p}=.043\right)$, 
so that the longer duration predicted higher sensitivity; a significant interaction between task and condition, to the effect that the dual-task cost on sensitivity was increased in the color task $\left(\square_{\text {motionXsingle }}=-1.13,95 \% \mathrm{CI}[-1.69,-0.57], \mathrm{t}=3.95, \mathrm{p}<.001\right)$.

The model for metacognitive efficiency had task, condition, and their interaction as fixed effects, and a random intercept for every participant. This model (intercept $=0.37$, participant $\mathrm{SD}=0.59$ ) had a significant effect of task $\left(\square_{\text {motion }}=-0.61,95 \%\right.$ CI $\left.[-0.82,-0.39], \mathrm{t}=5.63, \mathrm{p}<.001\right)$, describing participants' better metacognitive efficiency in the color task; a significant effect of condition $\left(\square_{\text {single }}=-0.43,95 \% \mathrm{CI}[-0.64,-0.22], \mathrm{t}=4.00, \mathrm{p}<.001\right)$, showing participants' overall worse metacognition in the single-task condition; and a significant task $\mathrm{X}$ condition interaction $\left(\square_{\text {motionXsingle }}=0.55,95 \% \mathrm{CI}[0.25,0.85], \mathrm{t}=3.62, \mathrm{p}<.001\right)$. 


\section{Supplementary Plots}

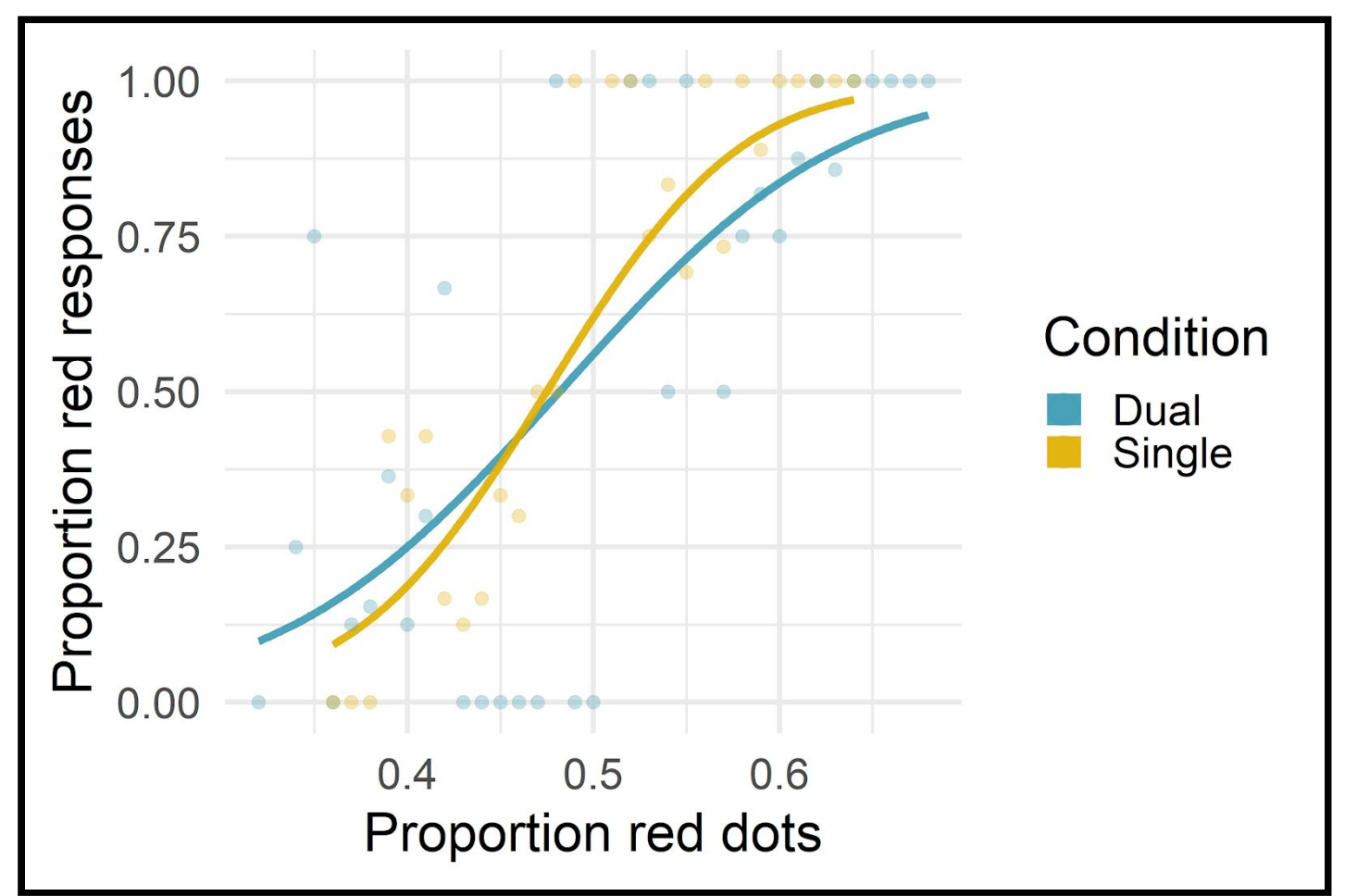

Figure F - Psychometric Fit of Discrimination Responses

The figure shows the psychometric fitted curves for one sample participant, describing the proportion of responses indicating "red" responses in the color task, as a function of the proportion of red dots relative to blue dots, in the two experimental conditions. The dots show the proportion of "red" responses, for different proportions of red VS blue dots. The curve was fitted through a cumulative gaussian function, with the quickpsy package in the $\mathrm{R}$ environment. 


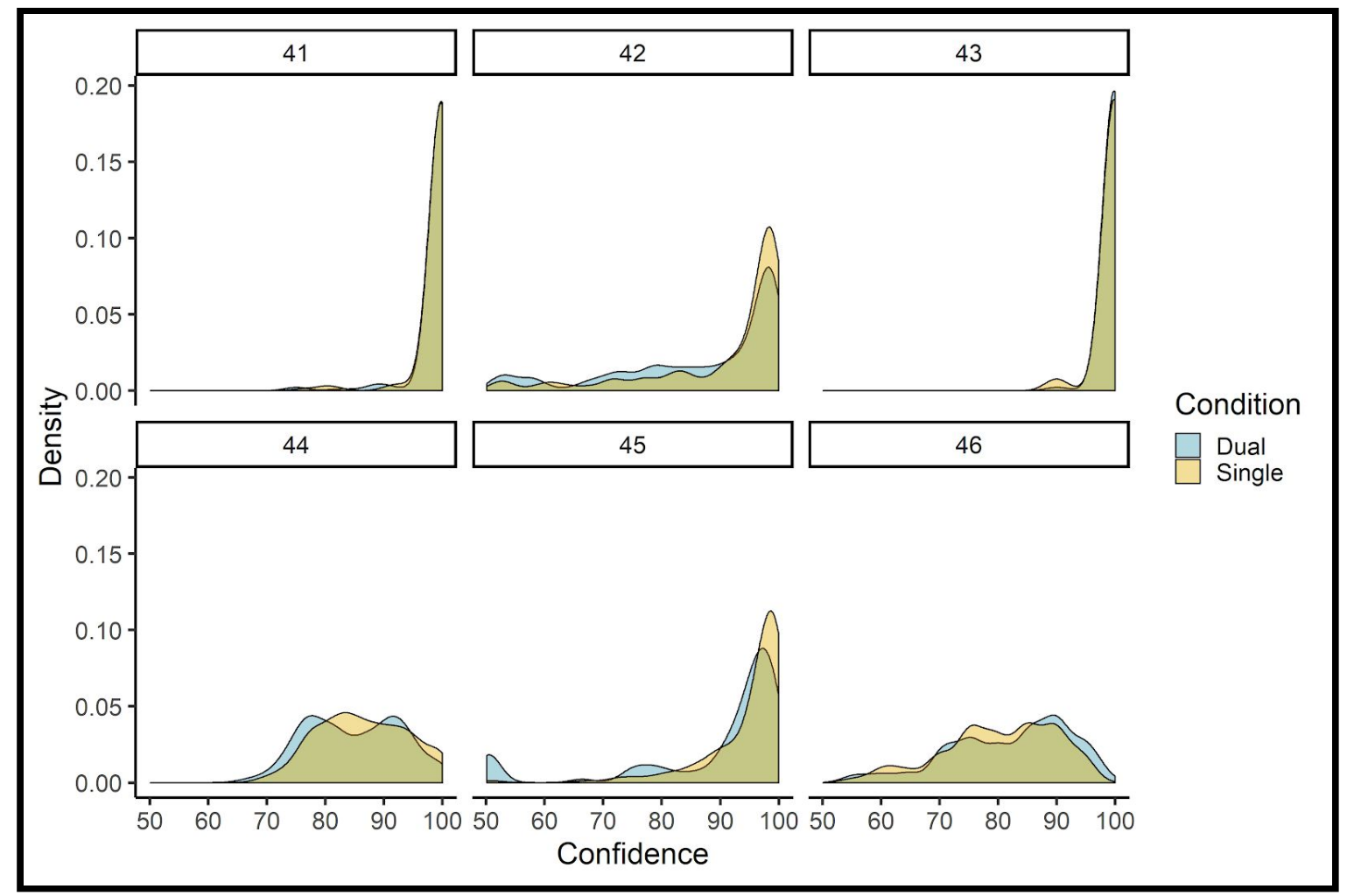

Figure G - Exclusion Criteria - Confidence Values

The plot illustrates the distribution of confidence values in one task (color), for both conditions, of six of our participants. Adopting our criteria, participants 41 and 43 are excluded from our analyses. 